UC-20

\title{
Reference Commercial Fusion Power Plants
}

by

J. R. Young

B. F. Gore

September 1976

Prepared for the Energy Research and Development Administration under Contract E(45-1)-1830 
NOTICE

This report was prepared as an account of work sponsored by the United States Covernment. Neither the United States nor the Energy Research and Development Administration, nor any of their employees, nor any of their contractors, subcontractors, or their employees, makes any warranty, express or implied, or assumes any legal liability or responsibility for the accuracy, completeness or usefulness of any imformation, apparatus, product or process disclosed, or represents that its use would not infringe privately owned rights.

\author{
PACIFIC NORTHWEST LABORATORY \\ operated by \\ BATTELLE \\ for the \\ ENERGY RESEARCH AND DEVELOPMENT ADMINISTRATION \\ Under Contract E(45-1)-1830 \\ PACIFIC NO
}
Printed in the United States of America Available from
National Technical Information Service
U.S. Department of Commerce
5285 Port Royal Road
Springfield, Virginia 22151

Price: Printed Copy \$5.30; Microfiche $\$ 2.25$ 
BNWL-2014

by

J. R. Young

B. F. Gore

September 1976

Battelle

Pacific Northwest Laboratories

Richland, Washington 99352 


\section{PREFACE}

Fusion reactor technology has developed far enough to expect laboratory demonstration of practical levels of fusion employing the $D-T$ reaction to occur in the early 1980s. Following that demonstration, and depending upon the national priorities for energy from $D-T$ fusion, construction and operation of experimental reactors and demonstration power reactors could occur before the end of this century. Operation of the first commercial power plants could then follow, starting about 2010 .

Development and adoption of a new power system eventually will require a description of the environmental effects in an environmental statement providing a comparison to the effects of competitive systems. In anticipation of that statement, an environmental analysis (BNWL-2010) has been prepared for the ERDA Division of Magnetic Fusion Energy. That analysis estimates the environmental effects of constructing and operating D-T fusion reactors as an economically competitive source of electricity in the 21 st century.

The analysis has four primary purposes:

1. To describe the general nature of the environmental effects,

2. To determine current ability to estimate the effects,

3. To determine methods for reducing the effects, and

4. To determine research necessary for increasing capability to define and reduce the effects.

Timely identification of needed research and methods for reducing effects will permit the performance of that research and the revision of conceptual fusion power plant designs before preparation of the program environmental statement. This would improve the quality of the environmental statements and could reduce the estimated adverse environmental effects due to fusion power plants.

The environmental analysis (BNWL-2010) concludes that the following assumed characteristics are the best set for the first operating fusion power plants:

- The D-T fusion reaction

- Large quantities of activation products

- Kilogram quantities of tritium in the plant systems

- Massive reactor structures

- Large lithium inventories

- Large inventories of liquid metals and salts
- Standard electricity generation

- Standard radioactive waste systems

- Large magnetic fields

- A self-contained fuel cycle

- Rural siting

Using these characteristics a reference reactor was analyzed to determine the environmental effects by using available concepts of plant subsystems designs that control interactions with the environment or by assumption that best current technology would be used in subsystems design.

Because this analysis does not take into account advances in both fusion and waste control technology during the next thirty years, the estimated effects probably are significantly higher than the actual effects will be for the first fusion power plants. The estimated environmental effects should be interpreted only as being the probable upper limit for the actual effects. 
Preparation of the fusion power plant environmental analysis required development and use of specially developed data and analysis methods not used in the preparation of current environmental statements for fossil and fission power plants. These data and analysis requirements are documented in a series of reference topical reports to make this information publicly available and to assure understanding of the basis for the conclusions made in the environmental analysis. These reference topical reports summarize the state-of-the-art as applicable to preparation of environmental statements for fusion power plants. They present the data and analytical techniques used in the environmental analysis to estimate the interactions with the environment and the resultant environmental effects. This information then was analyzed for adequacy and the need was determined for additional research to assure satisfactory ability to prepare environmental statements for the fusion development program and experimental facilities in the early 1980s. Estimated environmental effects are presented in these reference documents only as necessary to illustrate use of the data and analytical techniques.

This report is one of those reference documents for the environmental analysis. The other documents in this series contain more details of the power plant concepts and the probable environmental effects of fusion power plants with the assumed characteristics listed above. These documents are available through the National Technical Information Service:

An Environmental Analysis of Fusion Power to Determine Related R\&D Needs, BNWL-2010

Review of Fusion Research Program: Historical Summary and Program Projections, BNWL-2011

Fuel Procurement for First Generation Fusion Power Plants, BNWL-2012

Current Fusion Power Plant Design Concepts, BNWL-2013

Reference Commerical Fusion Power Plants, BNWL-2014

Siting Commercial Fusion Power Plants, BNWL-2015

Materials Availability for Fusion Power Plant Construction, BNWL-2016

Projected Thermodynamic Efficiencies of Fusion Power Plants, BNWL-2017

Tritium Source Terms for Fusion Power Plants, BNWL-2018

Management of Nontritium Radioactive Wastes from Fusion Power Plants, BNWL-2019

Methodology for Estimating Radiation Doses 0ue to Tritium and Radiocarbon Releases, BNWL-2020

Magnetic Field Considerations in Fusion Power Plant Environs, BNWL-202]

Biological Effects of iritium Releases from Fusion Power Plants, BNWL-2022

Biological Effects of Activation Products and Other Chemicals Released from Fusion Power Plants, BNWL-2023

Safety Review of Conceptual Fusion Power Plants, BNWL-2024

An Investigation of the Transportation Requirements of Fusion Power Plants, BNWL-2025

Considerations of the Social Impact of Fusion Power, BNWL-2026

Environmental Impacts of Nonfusion Power Systems, BNWL-2027

Environmental Cost/Benefit Analysis for Fusion Power Plants, BNWL-2028

Biomagnetic Effects: A Consideration in Fusion Reactor Development, BNWL -1973

An Analysis of Tritium Releases to the Atmosphere by a CTR, BNWL-1938 


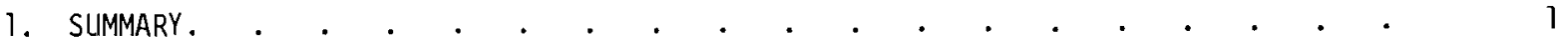

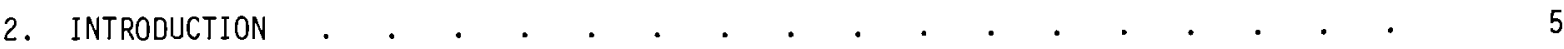

3. DEVELOPMENT OF THE ENVELOPE OF POWER PLANT CHARACTERISTICS . . . . . . $\quad$. 7

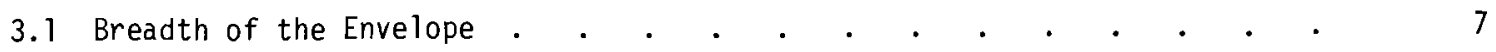

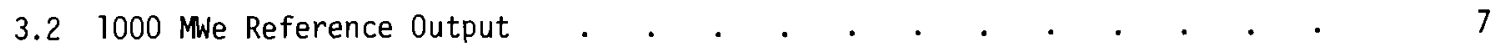

3.3 Linear Scaling of Size Dependent Parameters . . . . . . . . . 8

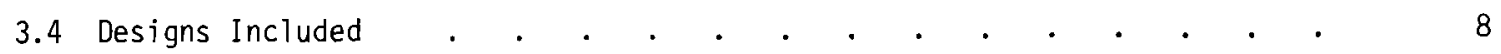

3.5 Functionally-Based Descriptive Format . . . . . . . . . . . . 9

4. THE ENVELOPE OF D-T FUSION POWER PLANT CHARACTERISTICS . . . . . . . $\quad$. 13

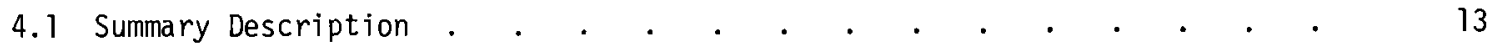

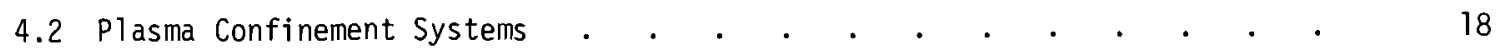

4.3 Plasma Heating Systems . . . . . . . . . . . . . . . . . . . . . . . 25

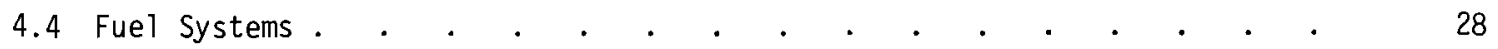

4.5 Blankets . . . . . . . . . . . . . . . . . . . . . . . . . . . 30

4.6 Heat Transfer Systems . . . . . . . . . . . . . . . . . . . 41

4.7 Electricity Generation Systems . . . . . . . . . . . . . . $\quad$. 44

4.8 Spent Plasma Collection Systems . . . . . . . . . . . . . . . . . 47

4.9 Bred Tritium Recovery Systems . . . . . . . . . . . . . . . . . . . 48

5. CHARACTERIZATION OF THE REFERENCE POWER PLANTS . . . . . . . . . . 51

5.1 Power Plant Environmental Interactions . . . . . . . . . . . 51

5.2 The Reference First Commercial Fusion Power Plant . . . . . . 56

5.3 The Reference Advanced Commercial Fusion Power Plant . . . . . 58

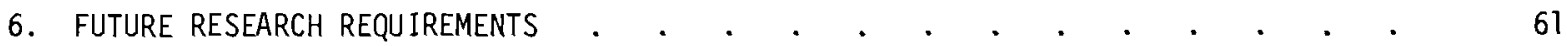

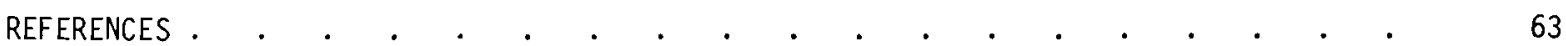




\section{LIST OF FIGURES}

Functional Relations Between Major Systems of D-T Fueled Fusion Power PIants

Fuel Loop Flow Diagram

Heat Transfer System Flow Schematic

Tokamak Toroidal Field and Central Support Structure

Tokamak Cross Section Showing Toroidal and Vertical Field Coils 20

Mirror Confinement of Plasma Between Yin Yang Field Coils 21

Cutaway View of Support Structure for Yin Yang Mirror Coils 21

Cutaway View of a Segment of a Theta Pinch Torus 22

Cross Section and Flow Schematic of the Wetted Wall Laser-Pellet Fusion Power Plant 24

Cross Section of the Blascon Pressure Vessel and Lithium Vortex 24

Neutral Beam Injection System Schematic

25

Section Through a Segmented Blanket Showing Ribbed First Wall Stressed in Tension

Sectional View of the First Wall and Radiation Shield of a Helium Cooled Blanket Containing Liquid Flibe

Blanket of Tubes Carrying Lithium Coolant Along Magnetic Field Lines

of the Mirror Confinement Design 


\section{LIST OF TABLES}

1 Originators of D-T Fueled Commercial Conceptual Power Plant Designs

2 Envelope of D-T Fusion Power Plant Characteristics 14

3 Typical Power Plant Environmental Interactions 52

4 Causes of Fusion Power Plant Environmental Interactions 57 


\section{SUMMARY}

Currently available conceptual designs for conmercial fusion power plants are for first generation plants using deuterium-tritium (D-T) fuel, and are a 11 functionally similar. This similarity has been used as a basis for defining an envelope of $D-T$ fusion power plant characteristics which encompasses the characteristics of the available designs. A description of this envelope, including general process descriptions, proposed materials uses and a tabulation of numerical ranges of plant parameters is presented in this document.

The envelope of $D-T$ fusion power plant characteristics has been analyzed to identify those characteristics which could cause significant environmental interactions in addition to interactions common to all thermal power plants. Those characteristics define a Reference First Commercial Fusion Power Plant.

No design concepts have been published for an advanced fusion power plant. Possible characteristics of a Reference Advanced Fusion Power Plant have been inferred from the envelope of current $D-T$ fusion power plant characteristics assuming that the fusion reaction employed neither creates nor consumes radionuclides.

These reference commercial fusion power plants provide the base for the Environmental Analysis of Fusion Power $(1)$ and its supporting documents. Identification of reference plant characteristics allows use of waste release systems not specific to any proposed design, and avoids the inference that any particular power plant concept is expected to be more successful than the others. Inclusion in the environmental analysis of all characteristics from the envelope ensures the conservatism of the environmental analysis, since environmental effects will be overestimated if actual commercial plants lack any of the included characteristics.

Characteristics of the Reference First Commercial Fusion Power Plant

Analys is of the characteristics of current fusion reactor design concepts indicates that the first commercial fusion power plants probably will have the following characteristics:

- The D-T Fusion Reaction

A11 current design concepts use this reaction because it is the first reaction expected to be achieved.

- Large Quantities of Activation Products

The D-T reaction creates large fluxes of $14 \mathrm{MeV}$ neutrons that will activate blankets, shields, coolants, and other materials in the main reactor system. However, no fission products or actinides are created.

- Kilogram Quantities of Tritium in the Plant Systems

Kilogram quantities of tritium will be fed into the reactor daily, and tritium will be created in the blanket regions to provide the reactor fuel. Because of its mobility this tritium will be widely dispersed throughout the plant. 


\section{- Massive Reactor Structures}

Heavy blankets and shields are necessary to moderate and capture the neutrons produced by the fusion reactions. They surround large plasma chambers which result from the low power density necessary to prevent excessive radiation damage of plasma chamber walls.

- Large Lithium Inventories

The tritium fuel is created by capturing neutrons in lithium. Large lithium inventories are needed to prevent capture of the neutrons by structural or shield materials.

- Large Inventories of Liquid Metals and Salts

Liquid metals and salts are used in most fusion reactor blanket concepts as primary and secondary coolants and for thermal energy storage.

- Standard Electricity Generation Systems

Most D-T fusion energy is released as neutron energy. That energy is converted to high temperature heat that creates steam to drive a standard turbine-generator to generate electricity.

- Standard Radioactive Waste Systems

Creation of tritium and activation products will require their containment, both during operation and maintenance outages. Standard gaseous, liquid, and solid radwaste systems will be necessary.

- Large Magnetic Fields

Most fusion power plant concepts include magnetic confinement of the fusion plasma. The resultant magnetic fields may extend for several hundred meters in all directions from the reactors.

- A Self-Contained Fuel Cycle

All fuel cycle facilities are included in the power plant. Only base fuel materials (deuterium, lithium, etc.) are delivered to the power plant and all products of the reaction are either vented, used in the process (helium), or buried as low-level radioactive solid waste.

- Rurar Siting

Because of the potential for radionuclide releases, exclusion areas comparable to those currently used for fission power plants probably will be necessary. Siting in urban areas does not appear practical.

Characteristics of the Reference Advanced Fusion Power Plant

Numerous fusion reactions are possible. Some reactions do not produce neutrons, deuterium, tritium, or other radionuclides. As a result, it may be possible to operate a fusion reactor that neither uses radionuclides as fuel nor produces radionuclides as reaction products or as a 
result of neutron activation of structural or coolant materials. (Although deuterium is not a radionuclide, creation of deuterium probably would result in secondary D-D reactions that produce neutrons and tritium.)

These cleaner fusion reactions require more severe operating conditions (temperature and plasma density) than are required for the D-T and D-D reactions. This will require more advanced technology than is needed for the reference first commercial fusion reactors.

Assuming that the required technology could become available, the advanced fusion reactor might have the following characteristics:

- A Clean Fusion Reaction

All primary fuels and reaction products would be nonradioactive materials. As an example, protium $\left({ }^{1} H\right)$ might be reacted with boron $\left({ }^{11_{B}}\right)$ to produce only helium and energy.

$$
{ }^{1} \mathrm{H}+{ }^{11} \mathrm{~B}+3^{4} \mathrm{He}+8.7 \mathrm{MeV} \text {. }
$$

- Minimal Radionuclide Production in the Power Plant

Some activation of structures by charged particles and radiation escaping from the plasma may occur. Side reactions in the plasma may also produce small quantities of radionuclides and neutrons which could activate structures.

- Moderate Reactor Structural Size

Blankets, shields, and massive concrete containment structures might not be needed. The reactor might be only a vacuum vessel surrounded by supporting equipment. Some shielding may be necessary to stop beta and gamma radiation.

- Standard and Advanced Electricity Generation Systems Normal conversion of heat energy to electricity probably would be required. Direct conversion equipment probably could be used.

- A Self-Contained Fuel Cycle

A11 fuel cycle facilities are included in the power plant. Only basic fuel materials (protium, boron, etc.) are delivered to the power plant, and all products of the reaction are either vented, used in the process (helium) or disposed of as nonradioactive solid waste.

- City Siting

Since the only waste products might be helium and heat, the environmental effects could be so low that the power plants could be operated in densely populated areas. 


\section{Research Requirements}

Development of these reference designs revealed that sufficient design detail has not been included in past descriptions of fusion power plant design concepts to permit preparation of a detailed reference design. To assure that future environmental analyses and statements for the fusion program describe the lowest practicable environmental impacts for the first commercial fusion power plants, a detailed reference design should be developed. In addition to the general reference design for the reactor as developed in this document, this reference design would include the following information:

1. Process flow diagrams showing the flow paths and rates of all major materials (fuels, coolants, wastes),

2. A description of the entire fuel cycle from mine to disposal of the wastes,

3. Sufficient descriptive material so that all radioactive inventories and their flow paths and rates are known,

4. A general description of all major supporting subsystems (waste treatment, ventilation, power generation),

5. Sufficient descriptions of any equipment that controls the release rates of pollutants so that the release rates can be determined,

6. Any unique siting or construction requirements, and

7. A tabulation of the quantities of each significant construction material showing location and degree of fabrication.

Future documents describing specific design concepts should also contain the above information. Research is needed in the development of individual plant designs, as discussed in BNWL-2013. Analysis of such designs to characterize reference fusion power plants is straightforward; therefore, research is not needed to improve the ability to characterize reference power plants. 


\section{INTRODUCTION}

Preparation of an environmental analysis requires availability of system designs on which to base the analysis. This document describes the two reference commercial fusion power plants used in preparation of the fusion reactor environmental analysis:

1. Reference First Commercial Fusion Power Plant

This first generation plant uses the deuterium-tritium fuel reaction, which requires the least stringent plasma conditions.

2. Reference Advanced Commercial Fusion Power Plant

This plant uses a fuel reaction for which neither fuel nor reaction products are radionuclides.

The characteristics of the reference commercial fusion power plants have been determined by analyzing currently available fusion power plant designs. Although years of research will be necessary before the actual designs of commercial fusion reactor power plants will be known, a number of design concepts have been proposed and several of these have been developed into fairly detailed designs. ${ }^{(2)}$ These concepts cover a broad range of possibilities, and probably include the general characteristics of commercial power plants necessary to establish a conservative envelope for estimating the upper limits of environmental effects.

Basing the environmental analysis on reference power plant characteristics minimizes the analysis efforts necessary to estimate the optimal performance achievable with best current technology of plant systems controlling interactions with the environment. Current fusion power plant designs were developed as part of the ERDA program to develop the technology of fusion, primarily to identify research and technology development needs. Design efforts were mainly devoted to systems necessary to induce fusion and recover the released energy. Many design documents lack descriptions of systems controlling the release of pollutants (e.g., radwaste systems). Systems which are described are generally not optimized. Use of reference fusion power plants removes the need to analyze the proposed design concepts separately.

The characteristics attributed to the reference comnercial fusion power plants include all with potential unique environmental interactions in comparison to current thermal power plants. Since actual commercial fusion power plants may not have all the environmental interactions attributed to the reference plants, the reference plants therefore represent environmental "worst cases." This ensures the conservatism of the environmental analysis by overestimation of environmental effects if actual commercial plants lack any of the included characteristics.

The currently available fusion power plant design concepts are all functionally similar. In each, a fusioning D-T plasma is confined, heated and fueled; neutron and radiation energy are captured and converted to heat in a blanket surrounding the plasma; the blanket is cooled and the heat is transported to a thermal-cycle generating system; and tritium is recovered from unburned plasma and from lithium-containing blanket materials, for recycle to the plasma. 
This functional similarity was used as a basis for defining an envelope of D-T fusion power plant characteristics. A description of this envelope, including general process descriptions, proposed material uses, and a tabulation of numerical ranges of plant parameters is presented in this document.

The envelope was then analyzed to identify plant characteristics which could cause significant environmental interactions. The primary interactions considered were: use of land, labor and materials; release of heat, chemicals, and liquids; production of noises, vibrations, odors and magnetic fields; disposal of gaseous and solid wastes, and of radionuclides; aesthetics, and material transportation. Characteristics expected to cause such interactions were identified, and the characteristics expected to cause significant unique environmental interactions were then attributed to the reference first commercial power plant.

No design concept has been published for an advanced fusion power plant. Consequently, possible characteristics of such a plant have been inferred from the envelope of current $D-T$ fusion power plant designs, using the assumption that the fusion reaction employed neither creates nor consumes radionuclides. Such a $\mathrm{plant}$ represents the ideal, clean power plant of the future. 


\section{DEVELOPMENT OF THE ENVELOPE OF POWER PLANT CHARACTERISTICS}

The functional similarity of the characteristics of the available designs has been used as a basis for defining an envelope of D-T fusion power plant characteristics. This section discusses the breadth of concepts included within the envelope, the normalization and scaling of size-dependent parameters, the power plant designs which have been analyzed to define the envelope, and the functional similarity of the designs (due to use of the D-T fusion reaction) which is the basis for development of the envelope.

\subsection{Breadth of the Envelope}

The scientific feasibility of fusion power production has not been demonstrated. At this time it is not possible to predict which of the confinement concepts may prove to be scientifically and economically feasible, and which may be developed to the point of commercial application. Designs are included for both the magnetic plasma confinement and inertial plasma confinement concepts.

The designs encompassed by this envelope are based upon different extrapolations of present technological capabilities. The designs are conceptual, as opposed to fully engineered. They represent first-attempts to develop designs which incorporate all aspects of fusion power plant performance and define systems to perform the necessary functions for power plant operation. An objective of many of the design studies was to identify, through the design attempt itself, components which cannot be realistically designed at the present time. Thus technology can be developed while plasma confinement research is being carried out, to be ready when it is needed. In summary, this envelope encompasses power plant designs which are:

- Based upon a variety of possible confinement concepts.

- Based upon differing extrapolations of present technological capabilities.

- Conceptual in nature.

Furthermore, the design characteristics encompassed by this envelope represent present estimates of possibilities for future plants. Consequently, this envelope does not represent a design goal for future studies. Future fusion power plant designs will be determined by the results of ongoing and planned research and development programs, experimental plant experience, revised environmental and safety standards, and other economic and societal factors. Future development of designs may require modification of the envelope and the analysis upon which it is based.

\subsection{MNe Reference Output}

The physics of plasma confinement requires Tokamak and theta pinch designs to be large, and to have large outputs. Mirror machines can be designed for a range of sizes; the mirror design study focused on a minimum investment demonstration plant. The confinement of laser-pellet microexplosions can be achieved in relatively small chambers, and current concepts call for many chambers to be clustered in power plants of intermediate output. The electrical outputs of the conceptual power plant designs on which this envelope is based range from 200 to 4100 megawatts 
electric (MWe). Due to this broad range of capability, some form of normalization of sizedependent parameters is imperative. Because the size variations are related to confinement concept, and since the successful concepts are unknown, normalization is chosen arbitrarily. The envelope is based on production of 1000 MWe. This is approximately the output of present commercial fission power plants and is close to the upper 1 imit within existing NRC regulations.

\subsection{Linear Scaling of Size Dependent Parameters}

The objective of the environmental analysis which this document supports is to examine the environmental implications of providing a large fraction of the electrical demand of the U.S. through fusion power plants. The production of large amounts of power by many fusion power plants, perhaps even by many types of power plants is implied. At present, the optimum size for a fusion power plant is not known. It is assumed that the designs available for the concepts currently being developed are near optimum from the standpoint of quantities of material used and amounts of environmental impact per unit of electricity produced. For the purpose of defining the envelope of parameters for the production of 1000 MNe of electrical power, the size-dependent parameters of power plant designs have been scaled linearly.

\subsection{Designs Included}

Conceptual designs for D-T fueled commercial power plants have been developed as part of the ERDA program to develop the technology of fusion. Designs have been developed based on each of the magnetic plasma confinement concepts under investigation (Tokamak, theta pinch and mirror) and for inertial plasma confinement concepts. As background for the environmental analysis for which this is a reference document, brief descriptions have been prepared of ten current designs. (2)

The ten fusion power plant designs are the starting point for the analysis of this document.

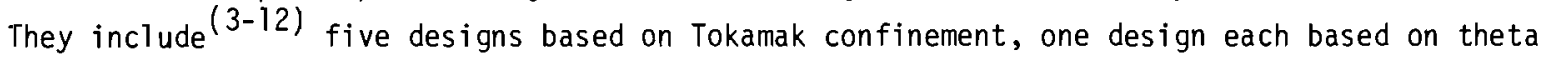
pinch and mirror confinement, and three designs based on inertial confinement. The designs were developed by study groups in the laboratories listed in Table 1.

TABLE 1 Originators of D-T Fueled Commercial Conceptual Power Plant Designs Magnetic Confinement

Tokamak

Theta Pinch

Mirror

Inertial Confinement

Blascon

Wetted Wall

Suppressed Ablation
University of Wisconsin [UWMAK-I (3) and UWMAK-II ${ }^{(4)}$ ] Brookhaven National Laboratory (BNL Blanket) (5) Princeton Plasma Physics Laboratory (PPPL) ${ }^{(6)}$ Oak Ridge National Laboratory (ORNL) ${ }^{(7)}$ Los Alamos Scientific Laboratory (LASL) $^{(8)}$ Lawrence Livermore Laboratory $(L L L)^{(9)}$ ORNL (10) $\operatorname{LASL}(11)$ LLL $(12)$ 


\subsection{Functionally-Based Descriptive Format}

All of the D-T fueled fusion power plant designs are functionally similar. In each, a fusioning D-T plasma is confined, heated and fueled; neutron and radiation energy are captured and converted to heat in a blanket surrounding the plasma; the blanket is cooled and the heat is transported to a thermal-cycle generating system; and tritium is recovered from unburned plasma and from lithium-containing blanket materials, for recycle to the plasma. This functional similarity allows system-based description and analysis of designs, which is the basis for development of the envelope of plant characteristics.

The description of the envelope of D-T fusion power plant characteristics is organized as follows:

- Summary Description

- Plasma Confinement Systems

- Plasma Heating Systems

- Fuel Systems

- Blankets

- Heat Transfer Systems

- Electricity Generation Systems

- Spent Plasma Collection Systems

- Bred Tritium Recovery Systems

Figure 1 shows functional relations between these major plant systems. Brief descriptions of the functions of these systems follow.

The Plasma Confinement System holds the fusioning plasma together at thermonuclear temperatures and densities long enough to release more fusion energy than is expended in creating the plasma, and confines unburned fuel and reaction products exiting from the burn region for subsequent recovery.

The Plasma Heating System delivers large amounts of energy to the plasma, thus heating it to the near-solar temperatures needed for fusion.

The Fuel System purifies recycled D-T fuel, prepares it and new fuel in the form necessary for use, and injects the appropriate quantity into the plasma chamber at the appropriate time and velocity.

The Blanket of material surrounding the plasma chamber performs many functions. It:

- Converts neutron energy to heat,

- Breeds tritium fuel by neutron interactions with lithium,

- Contains the fuel and other particles escaping from the plasma,

- Provides a vacuum chamber for the plasma (in some cases),

- Shields outer structures from fusion neutrons and other energy sources,

- Contains tritium bred within it, and

- Contains the coolant which transports heat from the blanket. 


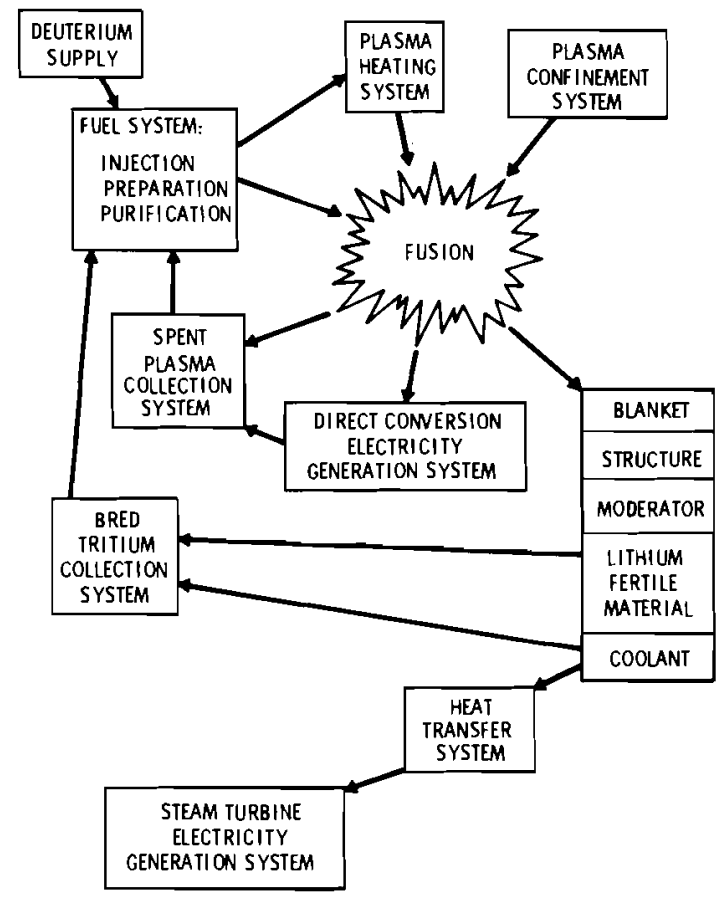

FIGURE 1 Functional Relations Between Major Systems of D-T Fueled Fusion Power Plants

The Heat Transfer System removes heat from blanket materials and transports it to the electricity generating system. It contains and circulates the coolants, and confines any radioactive materials that are contained therein.

Electricity Generation Systems convert heat energy to electricity using turbine-generators. In addition, they may directly convert the kinetic energy of charged particle motion into electricity without conversion to heat and the use of a thermodynamic cycle.

The Spent Plasma Collection System collects escaping plasma particles during and after the thermonuclear burn. Since only a small fraction of the fuel in the plasma is consumed, this system recycles collected plasma to the fuel system.

The Bred Tritium Recovery System recovers tritium bred by neutron interactions in blanket materials, and delivers it to the fuel system.

A fuel flow loop is central to the functional diagram of Figure 1. It functions to recycle unburned fuel escaping from the plasma to the fuel system for purification and reinjection into the plasma. Fuel can be injected into the plasma either directly, or through the plasma heating system. Makeup of consumed fuel is achieved by deuterium supply from outside the plant, and by the collection of tritium bred in the blanket. Helium ash from fusion and other plasma impurities are removed by the fuel system. Fuel flows are indicated in figure 2. 


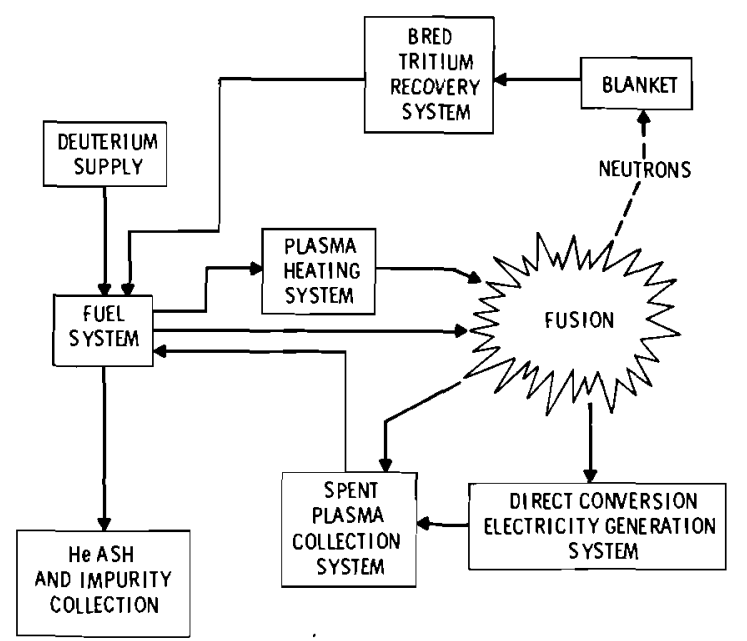

FIGURE 2 Fuel Loop Flow Diagram

A series of coolant flow loops is used to transport heat energy from the blanket to drive the turbine-generators. These loops are indicated in the heat transfer system flow in Figure 3 . In some cases an intermediate coolant loop is not included.

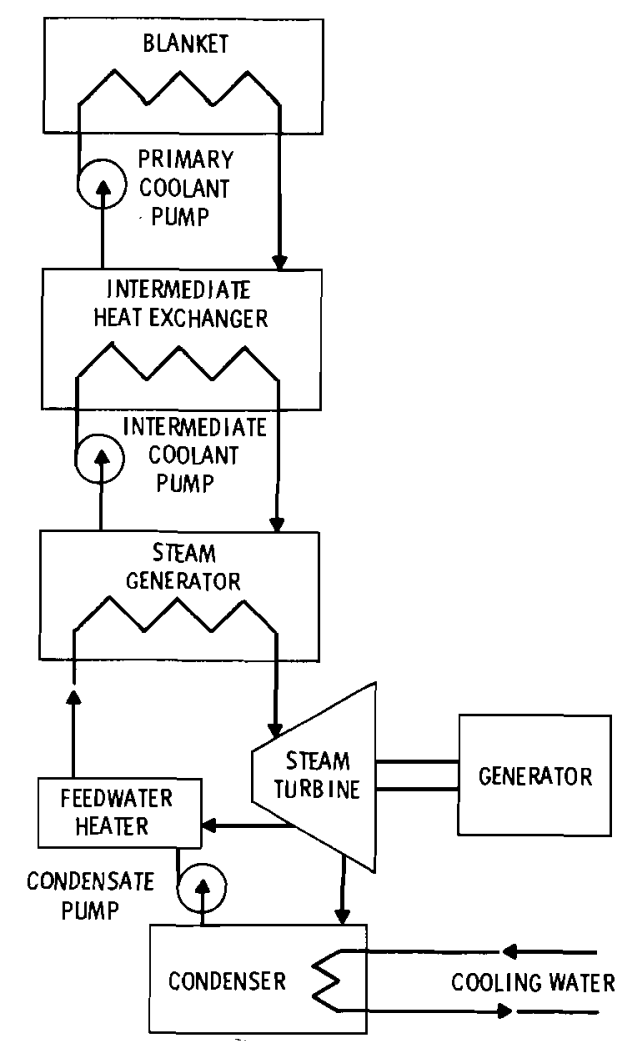

FIGURE 3 Heat Transfer System Flow Schematic 


\section{THE ENVELOPE OF D-T FUSION POWER PLANT CHARACTERISTICS}

A description of the envelope, including general process descriptions, proposed material uses, and a tabulation of numerical ranges of plant parameters is presented in this section.

\subsection{Summary Description}

The envelope of D-T fusion power plant characteristics encompasses power plant designs linearly scaled to the production of 1000 MWe of net electrical power. Principal plant parameters, and the ranges of their values encompassed by the envelope are listed in Table 2.

Generation of the reference 1000 MWe of electrical power is accomplished from a thermal energy release ranging from 1930 to 3800 megawatts thermal. Generation is continuous for all but two Tokamak designs considered which require periodic interruptions every three minutes to 1-1/2 hours depending on design.

Primary coolants encompassed by the reference envelope are liquid lithium metal, and helium gas. Low radioactive intermediate coolants are specified to separate liquid lithium from steam systems. Storage of high temperature intermediate coolants for circulation to steam generators during hourly reactor shutdowns is used in some Tokamak designs to allow continuous electrical generation despite the cyclic reactor operation.

Containment is provided by appropriately designed structures which enclose the fusion reactors and associated process systems containing radioactive materials. Evacuated regions, doublewalled piping, inert atmospheres and purge flows provide additional safety and containment features.

Reactor diameters range from 30 to 43 meters for magnetic confinement designs, except for the 115 meters diameter theta pinch reactor. Inertial confinement reactor chambers range from 6 to 8 meters in diameter, with many chambers required in plants producing the reference 1000 MWe output. Tokamak and mirror magnetic confinement of fusioning plasma is achieved by superconducting magnets of $\mathrm{NbTi}$ or $\mathrm{Nb}_{3} \mathrm{Sn}$ superconductor stabilized with copper. These designs require plasma region magnetic fields ranging from 2.5 to 6 tesla. The pulsed theta pinch magnet coils are made of copper and produce fields in the plasma chamber of 11 tesla. Plasma chamber walls confine spent plasma after the thermonuclear burn in both magnetic and inertial confinement devices.

Magnetically confined plasma is heated by megampere plasma currents in Tokamak devices. Energetic beams of neutral fuel atoms carrying tens to hundreds of megawatts of power are injected also in Tokamaks, and in mirror devices. Plasma is heated in theta pinch devices by magnetic implosion and compression.

In inertial confinement designs solid fuel pellets are imploded and heated to thermonuclear ignition by laser beams which deliver between 0.1 to 1.0 megajoules of energy to millimetersized pellets. Simultaneous pellet irradiation by multiple laser beams is required, at laser pulse rates between 6 and 40 times per second.

Fuel consumption due to thermonuclear reactions ranges from 0.23 to 0.47 kilograms per day $(\mathrm{kg} /$ day) of tritium and from 0.15 to $0.31 \mathrm{~kg} /$ day of deuterium, depending on the thermal efficiency of the device. Because only a fraction of the plasma reacts before escaping 
TABLE 2 Envelope of D-T Fusion Power Plant Characteristics

Overall Performance

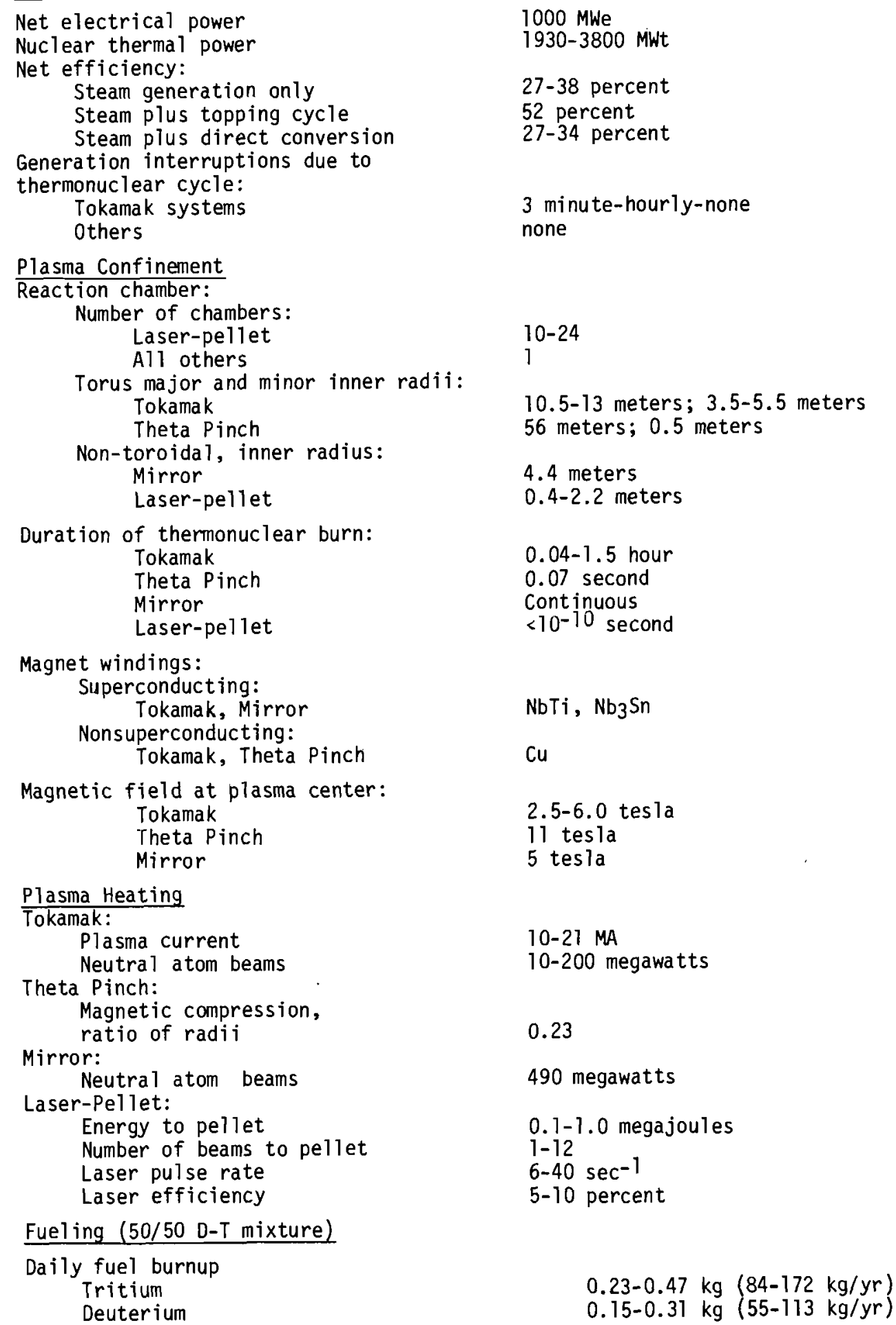




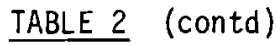

Injected fuel form:

Tokamak, Mirror

Theta Pinch

Laser-Pellet

Daily fuel injection

Tritium

Deuterium

Percent burnup

Blanket

Inner wall:

Neutron energy loading

Thermal energy loading

Total flux

Replacement interval

Materials:

Structure

Coolant

Tritium Breeding

Neutron moderating

Neutron multiplying

Shielding

Primary conceptual combinations:

Combination \#1

Combination \#2

Combination \#3

Blanket thickness:

Magnetic confinement

Laser-pellet

Shield thickness (magnetic conf.)

Heat Transfer

Major coolant chain combinations:

Combination \#1

Combination \#2

Combination \#3

Number of parallel loops

Primary coolant system

Hot leg temperature:

Li coolant

SS structure

Nb-Zr structure

Hot leg temperature:

He coolant

Pe-16 structure; f1 ibe

316 SS structure; solid

Li compounds

$6060 \mathrm{AT}$ (insulated) struct; solid Li comp.

Hot leg-cold leg $\Delta T$ :

Li coolant

Magnetic confinement designs He coolant Laser-pellet designs

$\mathrm{L} i / \mathrm{Na}$, NaK, $\mathrm{K}$ or He/Steam

$\mathrm{He} / \mathrm{Na} / \mathrm{Steam}$

$\mathrm{He} / \mathrm{Steam}$

$\mathrm{T}-12$

$0.4-6.7 \mathrm{MW} / \mathrm{m}^{2}$

$1-11 \mathrm{MW} / \mathrm{m}^{2}$

$2-27 \times 10^{14} \mathrm{~cm}^{-2} \mathrm{sec}^{-1}$

$2-10$ years

316 SS; PE-16; $6061 \mathrm{Al} ; \mathrm{Nb}-\mathrm{Zr}$

Li metal; He gas

Li metal; flibe; LiAl; LiAl02

(natural or ${ }^{6} L i$ enriched)

$C$; Be; flibe; $L i$ compounds

Be; ${ }^{2} \mathrm{i} ;$ flibe

$\mathrm{Pb} ; \mathrm{B}_{4} \mathrm{C}$; SS; Hydrocarbons;

Concrete

Liquid Li moderator and coolant

Molten flibe moderator;

He coolant

Solid Li compound moderator;

He coolant

0.4-1.0 meters

1. 0-3.8 meters

0.5-1.1 meters

$490-550^{\circ} \mathrm{C}$

$550-1050^{\circ} \mathrm{C}$

$640^{\circ} \mathrm{C}$

$650^{\circ} \mathrm{C}$

$760^{\circ} \mathrm{C}$

$56-130^{\circ} \mathrm{C}$

$100-800^{\circ} \mathrm{C}$

$200-550^{\circ} \mathrm{C}$ 
TABLE 2 (contd)

Mass Flow (Total)

Li coolant

Magnetic confinement designs

Laser pellet designs

He coolant

Intermediate coolant system

Hot leg temperature:

$\mathrm{Na}$ coolant

$\mathrm{K}$ binary topping cycle

Hot leg-cold leg $\Delta \mathrm{T}$ :

$\mathrm{Na}$ coolant

$\mathrm{K}$ boiler

Mass flow:

$\mathrm{Na}$ coolant

Steam system

$K$ coolant (boiling)

Main steam temperature

Mass flow (total)

Feedwater temperature

Spent Plasma Collection

Required vacuum pumping speed:

Tokamak with divertor

Theta-Pinch

Mirror with direct convertor

Laser-pellet

$2.0-6.2 \times 10^{7} \mathrm{~kg} / \mathrm{hr}$

$0.3-1.2 \times 10^{7} \mathrm{~kg} / \mathrm{hr}$

$2.6-8.8 \times 10^{6} \mathrm{~kg} / \mathrm{hr}$

$455-570^{\circ} \mathrm{C}$

$982^{\circ} \mathrm{C}$

$100-245^{\circ} \mathrm{C}$

$55^{\circ} \mathrm{C}$

$3.0-8.1 \times 10^{7} \mathrm{~kg} / \mathrm{hr}$

$3.5 \times 10^{6} \mathrm{~kg} / \mathrm{hr}$

$400-565^{\circ} \mathrm{C}$

$2.4-5.1 \times 10^{6} \mathrm{~kg} / \mathrm{hr}$

$220-320^{\circ} \mathrm{C}$

$10^{5}-10^{8} 1 / \mathrm{sec}$

$5.6 \times 10^{5} 1 / \mathrm{sec}$

$3.3 \times 10^{8} 1 / \mathrm{sec}$

$10^{4}-10^{6} 1 / \mathrm{sec}$

Bred Tritium Recovery

Tritium breeding ratio

Methods of $T$ collection

From liquid Li:

Option \#1

Option \#2

option \#3

Option \#4

From flibe:

From solid Li compounds:

Option \#1

option \#2

Residual $T$ concentration achievable:

In 1 iquid $\mathrm{Li}$

In flibe

In solid $\mathrm{Li}$ compounds

$1.0-1.5$

Gettering by solid or 1 iquid Gettering from intermediate coolant Permeation to vacuum system Cold trapping and filtering Spray evolution of TF

Diffusion; collection plenna

Diffusion to coolant He; oxidation; drying

$0.5-5 \mathrm{ppm}$

$0.2 \mathrm{ppm}$

$1.5 \mathrm{ppm}$

Tritium Inventories

Total plant:

Liquid Li designs

Flibe design

Solid Li designs

$1-16 \mathrm{~kg}$

$1.3 \mathrm{~kg}$

$6-10 \mathrm{~kg}$

Blanket and coolant:

Liquid Li designs

Flibe design

Solid Li designs

$0.1-6 \mathrm{~kg}$

$0.02 \mathrm{~kg}$

$0.01-0.3$

$T$ recovery system:

Liquid Li designs

Flibe design

Sol id Li designs

$0.1-3.1 \mathrm{~kg}$

$0.004-2.3 \mathrm{~kg}$ 
$\underline{\text { TABLE } 2}$ (contd)

\begin{tabular}{cl} 
Fuel system: & \\
Liquid $L i$ designs & $0.2-0.4 \mathrm{~kg}$ \\
Flibe design & $0.25 \mathrm{~kg}$ \\
Solid Li designs & $0.07 \mathrm{~kg}$ \\
Storage & \\
Liquid Li designs & $0.4-10.8 \mathrm{~kg}$ \\
Flibe design & $1.0 \mathrm{~kg}$ \\
Solid Li designs & $6.2-7.5 \mathrm{~kg}$ \\
Solid Waste Production (Total) & \\
\hline Blanket Structure/content combinations: & \\
SS/Li (liq) & $90-490$ metric tons per year \\
SS/Li (solid) & 675 metric tons per year \\
PE-16/Flibe & 115 metric tons per year \\
Nb/Li (liq) & $55-240$ metric tons per year \\
Al/Li (solid) & 285 metric tons per year
\end{tabular}

confinement (from 5 to 25 percent), rates of fuel injection to the plasma range from 1.2 to $7.4 \mathrm{~kg} /$ day of tritium and from 0.8 to $5.9 \mathrm{~kg} /$ day of deuterium. Methods of fuel injection include neutral atom beams, solid pellets accelerated either pneumatically or electrostatically, and straightforward metering of neutral fuel gas into the plasma chamber between burn cycles. Energy collection and tritium breeding take place in the blanket which surrounds the plasma chamber. Structural rigidity is provided by austenitic stainless steels, the nickel alloy PE-16, niobium, or an aluminum alloy. Tritium breeding is accomplished in molten lithium metal, the molten salt flibe $\left(L i F-B e F_{2}\right)$, the solid alloy $L i A l$, and/or the solid compound $L i A l O_{2}$. Natural lithium, lithium enriched in the isotope ${ }^{6} \mathrm{Li}$, or both, are used. Additional materials including beryllium and graphite may be included for neutron multiplication and moderation. Liquid lithium or helium gas are circulated through the blanket as the primary coolant.

Power plant performance depends strongly on blanket design features, as influenced by materials properties including high temperature strength, chemical compatibility, and physical location.

Major combinations of blanket structural material, fertile material, and coolant include: stainless steel structure with liquid lithium fertile material/coolant; and with helium cooled solid lithium fertile material; niobium structure with liquid lithium fertile material/coolant; PE-16 structure with helium cooled flibe fertile material and water cooled aluminum structure with helium cooled solid lithium fertile material.

Most of the fusion energy is deposited in the blanket and removed by the primary coolant at a high temperature. Particles and radiation penetrating the blanket are absorbed by additional shielding outside the blanket. Shield design and cooling depend upon radiation levels and spatial requirements, including those posed by superconducting magnets. Proposed shield materials include stainless steel, lead, boron carbide, hydrocarbons, and concrete.

Heat is transferred from the primary coolant to a steam system for the generation of electricity. Hot leg temperatures for liquid lithium primary coolants range from 914 to $1020^{\circ} \mathrm{F}\left(490\right.$ to $550^{\circ} \mathrm{C}$ ) 
in stainless steel structures and from 1020 to $1950^{\circ} \mathrm{F}\left(550\right.$ to $\left.1050^{\circ} \mathrm{C}\right)$ in niobium structures. Hot leg temperatures for helium primary coolants range from 1184 to $1400^{\circ} \mathrm{F}\left(640\right.$ to $\left.760^{\circ} \mathrm{C}\right)$.

Intermediate coolants between lithium and steam systems preclude dispersal of radioactive materials in the lithium in the event of steam generator leakage. Intermediate coolants include liquid sodium and NaK (sodium-potassium alloy), potassium in a liquid/vapor topping cycle, and helium. Periodic accumulation and release of sodium intermediate coolant, used with both lithium and helium primary coolants, allows continuous generation despite periodic brief reactor shutdowns. In other designs steam is generated directly from helium primary coolant.

Steam to drive the turbine generators is produced with temperatures ranging from 750 to $1050^{\circ} \mathrm{F}$ $\left(400\right.$ to $\left.565^{\circ} \mathrm{C}\right)$. Steam flows range from $2.4 \times 10^{6} \mathrm{kilograms}$ per hour $(\mathrm{kg} / \mathrm{hr})$ to $5.1 \times 10^{6} \mathrm{~kg} / \mathrm{hr}$. Electrical generation is primarily by conventional steam turbine generators, although direct conversion of plasma kinetic energy to electricity is also used in the theta pinch and mirror designs.

Plasma chambers are all evacuated to some degree, and vacuum pumps are used to collect spent plasma in many designs. Entrainment and absorption of unburned fuel in liquid lithium is also used. Vacuum pump speeds range from $10^{4}$ to $10^{8}$ iiters per second.

Bred and absorbed tritium is recovered from liquid lithium, flibe, or solid lithium fertile materials. Tritium is removed from liquid lithium by solid and liquid gettering agents, by permeation into an intermediate coolant or into the vacuum system, and by filtered cold traps. Gettering agents include yttrium metal foil and a mixture of the molten salts KCl and LiC1. Liquid lithium is used as a gettering agent in intermediate coolants, as is yttrium metal foil. Tritium bred in flibe is present as tritium fluoride, which is evolved from droplets sprayed into an evacuated tank. Tritium diffusing from solid lithium compounds enters special collection plenna, or is entrained in helium coolant passing through beds of the fertile material. Tritium is recovered from helium coolant by oxidizing it and absorbing the resulting tritiated water on beds of drying agent which are periodically regenerated.

Additional information on the envelope is presented in Table 2, including tritium inventories of plant systems, and quantities of solid waste produced by replacement of radiation damaged blanket structures.

\subsection{Plasma Confinement Systems}

Various magnetic and inertial confinement schemes have been proposed for use in fusion power plants. They hold the fusioning plasma together at thermonuclear temperatures and densities long enough to release more fusion energy than is expended in creating the plasma, and confine unburned fuel and reaction products exiting from the burn region for subsequent recovery and reprocessing. They differ in the need for magnet systems, the duration of thermonuclear burn, and the method of protecting the plasma chamber wall.

Magnetic Confinement - The magnetic confinement of thermonuclear plasma is difficult, requiring complex systems and geometries. The configuration of the confining magnetic field is so 
fundamental to the design that it primarily determines the configuration of the reactor. The locations of the magnet coils which produce the confining magnetic fields, and the necessity of shielding these coils from neutrons and radiation, also influence reactor design.

Tokamak Magnetic Confinement - Tokamak fusion power plants require four types of magnet coils. A toroidal magnetic field is produced by large bore magnets, shown in Figure 4, which encircle the plasma chamber and blanket. These toroidal magnets will be superconducting, to minimize power dissipation, and will require large amounts of normal conductor material for cryogenic stability. They will also require massive structural supports to resist both deformation and dislocation by magnetic forces.

In addition to a toroidal magnetic field, plasma confinement in Tokamak fusion power plants requires vertical magnetic fields induced by large horizontal coils lying parallel to the toroidal ring of plasma. These fields are pulsed on for the thermonuclear burn, which may last from several minutes to more than an hour; then they are shut off during the intervening reactor recharge period. The toroidal field coils operate continuously. The following paragraphs describe the various coils which produce vertical fields, and their functions.

Magnet coils producing vertical fields in Tokamak fusion power plants may or may not be superconducting, depending on economic, maintenance and structural considerations. They are illustrated in Figure 5. The large size of Tokamak magnet coils may be seen in the figure by comparing them to the two-meter man drawn at the base of the machine.

In Tokamak devices the circular loops of the toroidal magnetic field are distorted into helices. This is achieved by inducing a plasma current of millions of amperes via transformer action. The coils primarily responsible for this function are called "transformer" or "ohmic heating" coils (the plasma current also heats the plasma).

The radial and vertical position of the plasma ring is determined by vertical magnetic fields; coils primarily responsible for this function are sometimes called "vertical field" coils. The

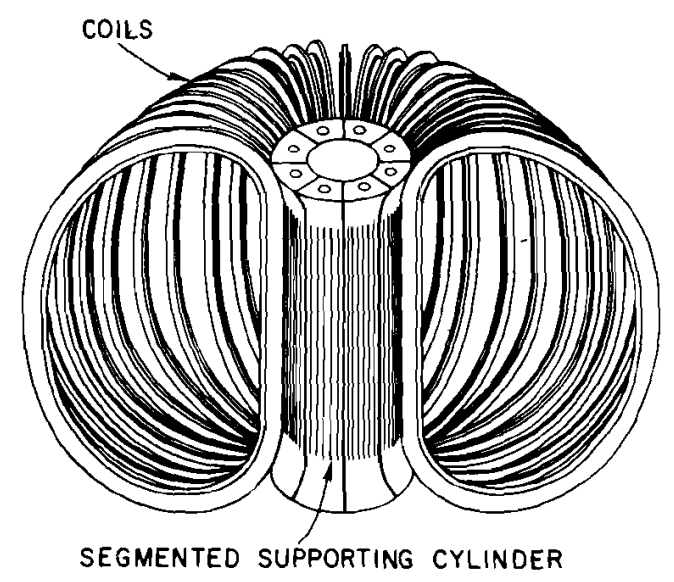

FIGURE 4 Tokamak Toroidal Field and Central Support Structure 


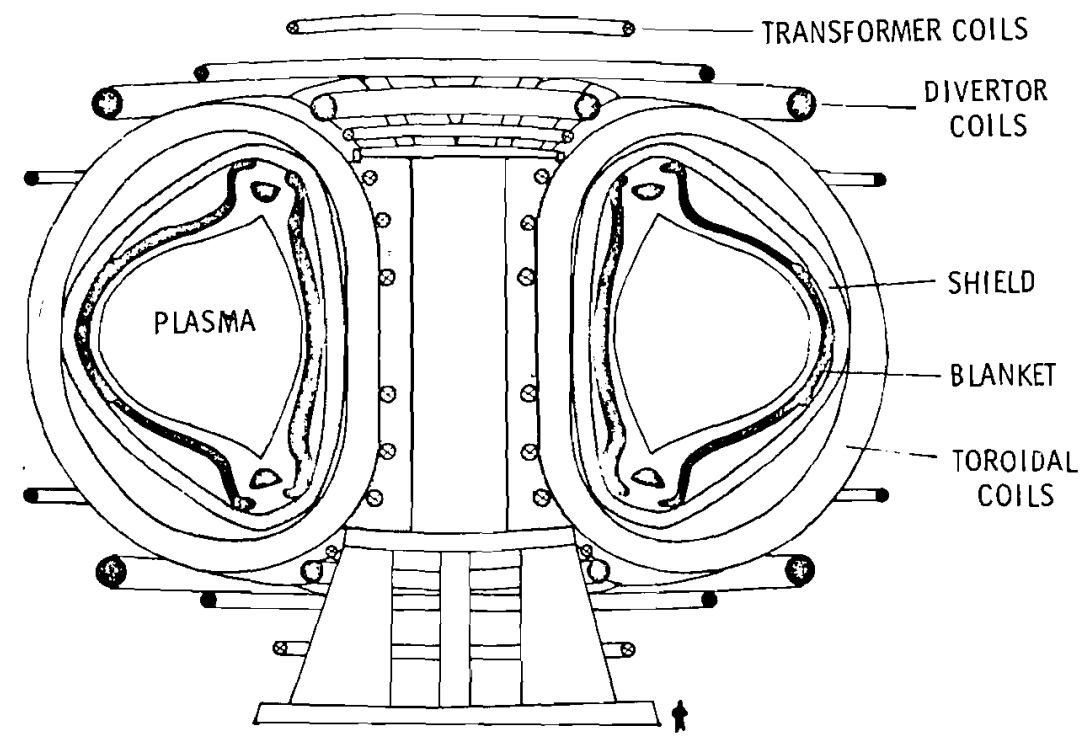

FIGURE 5 Tokamak Cross Section Showing Toroidal and Vertical Field Coils

magnetic field of "diverter" coils, in combination with the other fields, may be used to guide charged particles escaping from the plasma into special collection chambers. This facilitates collection, and protects the plasma chamber walls. Relaxation of these vertical fields at the termination of the thermonuclear burn must be carefully programmed to maintain protection of the plasma chamber walls until the plasma energy is dissipated.

Mirror Magnetic Confinement - Only portions of magnetic field loops are contained within the plasma chamber of a mirror fusion reactor. "Mirror" regions of high magnetic field reflect charged particles spiraling along field lines. The plasma is trapped between two such mirror regions. Mirror confinement of plasma between "Yin Yang" magnet coils is illustrated in Figure 6. The magnetic field lines passing through each of the coils are also illustrated, along with the trapped plasma. The regions of mirror reflection occur where the field lines pass through each coil.

Mirror confinement is inherently leaky. Charged particles following a field line sufficiently close can penetrate a mirror and collisions continually scatter plasma particles into such directions. However, operation of mirror fusion reactors is expected to continue due to plasma replenishment by injection of energetic beams of neutral fuel atoms. Plasma particles escaping through the mirror regions are channeled to a collection region which may be a direct conversion system for the generation of electricity.

The Yin Yang magnets are expected to be superconducting, and will require large amounts of superconductor and normal conducting materials. The large forces tending to distort and displace the magnet coils will be resisted by hoop stresses in a surrounding spherical structure shown in Figure 7. The large size of the magnets and structure is illustrated by comparing them to the two-meter men in the foreground of the figure. 

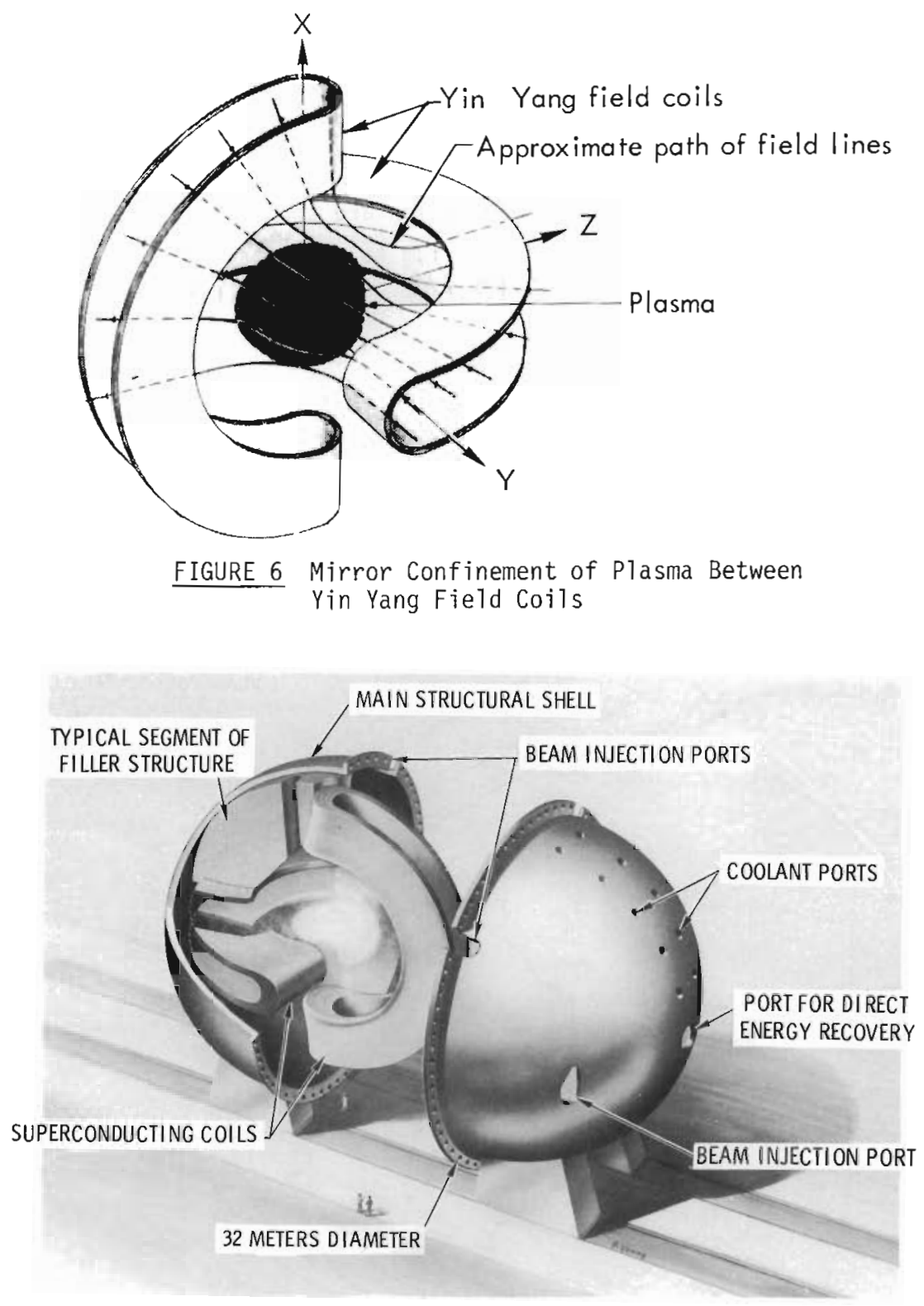

FIGURE 7 Cutaway View of Support Structure for Yin Yang Mirror Coils

Theta Pinch Magnetic Confinement - In a theta pinch device, fast-rising magnetic fields are used to heat and compress plasma to thermonuclear ignition conditions. Applied magnetic fields induce currents in the plasma by transformer action. These currents flow in the "theta" direction encircling the plasma ring. Interaction of these currents with the rising magnetic field heats and compresses the plasma - hence, the "theta pinch."

Theta pinch devices may operate in either linear or toroidal configurations; however, the toroidal configuration is preferred to prevent plasma loss out of the coil ends. The existing 
conceptual design specifies a torus major diameter of 112 meters. The minor diameter, including plasma chamber, reactor blanket and magnet coils, is only 3 meters. Thus, in contrast to Tokamak reactors, which are proportioned like fat doughnuts, a theta pinch reactor is expected to resemble a bicycle tire.

In Figure 8 a segment of a theta pinch torus is illustrated. The copper adiabatic compression coils responsible for plasma confinement and compression are shown encircling the blanket structure. These are in turn encircled by rings of titanium to support the large forces engendered by magnetic field interactions. Also shown is an inner magnet coil used to shock-heat the plasma prior to compression.

The operational mode of a theta pinch reactor will be pulsed, with the thermonuclear burn lasting less than a tenth of a second. The plasma expands against the confining magnetic field

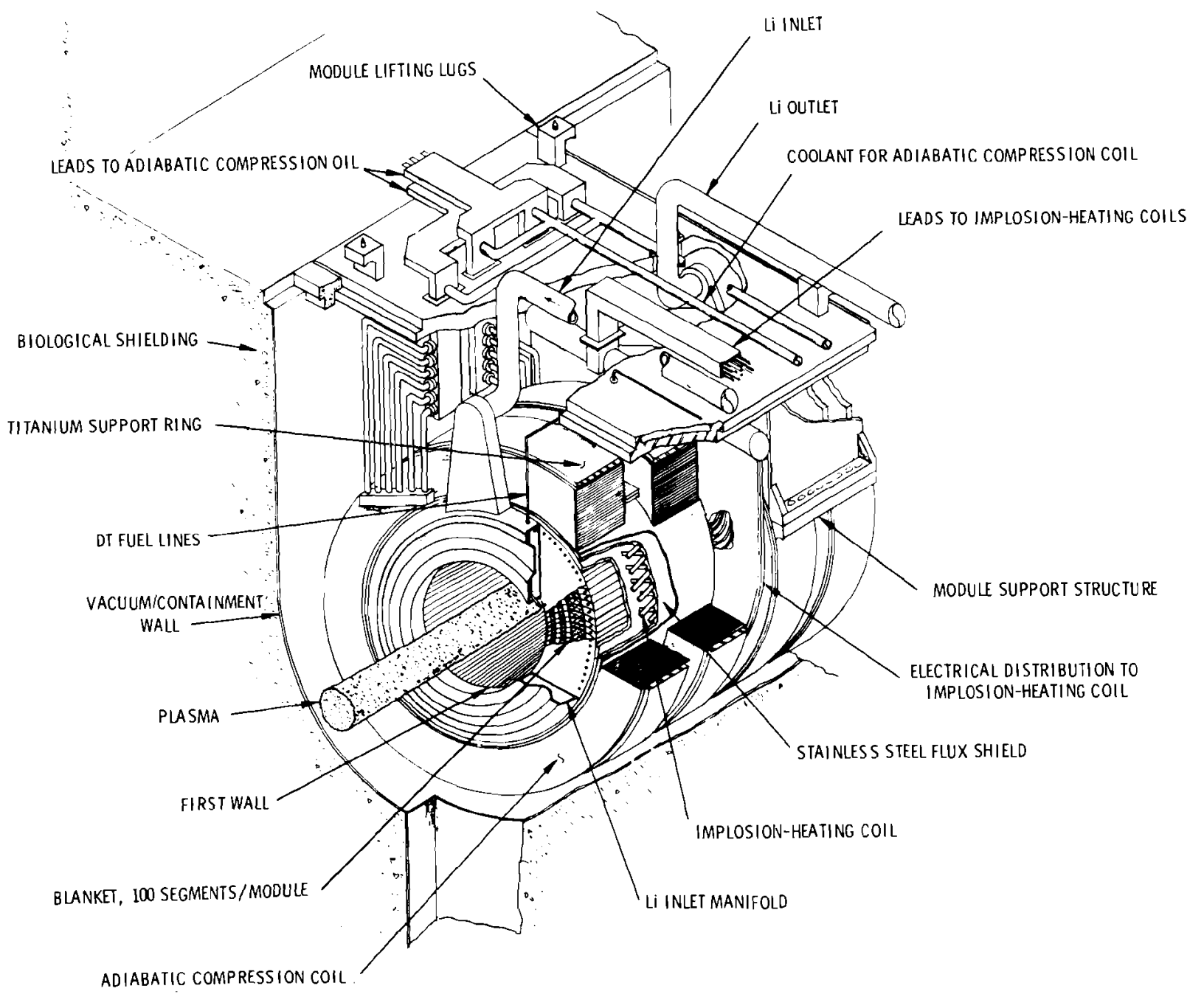

FIGURE 8 Cutaway View of a Segment of a Theta Pinch Torus 
during the thermonuclear burn, and the burn is terminated before the plasma contacts the plasma chamber wall. In addition to the confining magnetic field, further wall protection is provided by the introduction of neutral fuel gas between the plasma and the wall during the burn. This gas helps to transfer the plasma energy to the wall as the plasma is quenched and neutralized at the termination of the burn.

Inertial Confinement - Inertial confinement is actually nonconfinement. Following compression and heating of a fuel pellet by laser beams, the thermonuclear plasma disassembles on a time scale determined by plasma particle velocities - fractions of a nanosecond. Initial plasma conditions alone must ensure that an adequate thermonuclear burn takes place before the plasma disassembles. Thus, laser-pellet fusion power plants lack systems to confine the plasma in the region of thermonuclear burn.

The debris from the thermonuclear microexplosions propagates to the reaction chamber wall. Although roughly three quarters of the energy of a fusion pulse is carried by neutrons, which distribute their energy over a volume of material, the remainder is delivered to the containment surface as the kinetic energy of pellet debris and helium ash, and as $x$-rays. Due to the extremely short duration of the thermonuclear burn the deposition rate of this energy is very high.

The three laser-pellet power plant designs used in defining the model plant reference envelope utilize different methods to protect the reaction chamber from very high rates of energy deposition. All three of them utilize a first surface of liquid lithium metal to receive the particles and radiation emanating from the fusion microexplosions.

Because the energy of particles and radiation emanating from fusion microexplosions is deposited within a fraction of a millimeter of a lithium surface, two of the designs propose surface coatings of 1 millimeter or less of liquid lithium. They differ in the amount of lithium vaporized from the surface, by varying the microexplosion energy release and the surface area of the reaction cavity. The significance of this variation is that ablative blowoff of the surface can cause shock waves which are orders of magnitude larger than those due to the impact of microexplosion debris.

Figure 9 shows a cross section of a reactor cavity proposed in the Wetted Wall conceptual design. Kilogram quantities of lithium are vaporized from the cavity surface by each fusion pulse. Pellet debris and lithium vapor undergo blowdown through a diffuser/condenser and are absorbed into a pool of liquid lithium. Confinement stresses are resisted by a thick structura 1 vessel located deep in the blanket.

Ablation of the lithium layer coating the surface of the reactor chamber is avoided in the Suppressed Ablation design concept by reducing the microexplosion energy release and increasing the reaction chamber surface area. Not only is the chamber enlarged, but its inner surface is lined with inward-projecting pyramids to further increase its area. Due to the reduction in structural stresses due to fusion microexplosions, chamber wall structural requirements are determined by neutronic and heat transfer requirements in this design. 


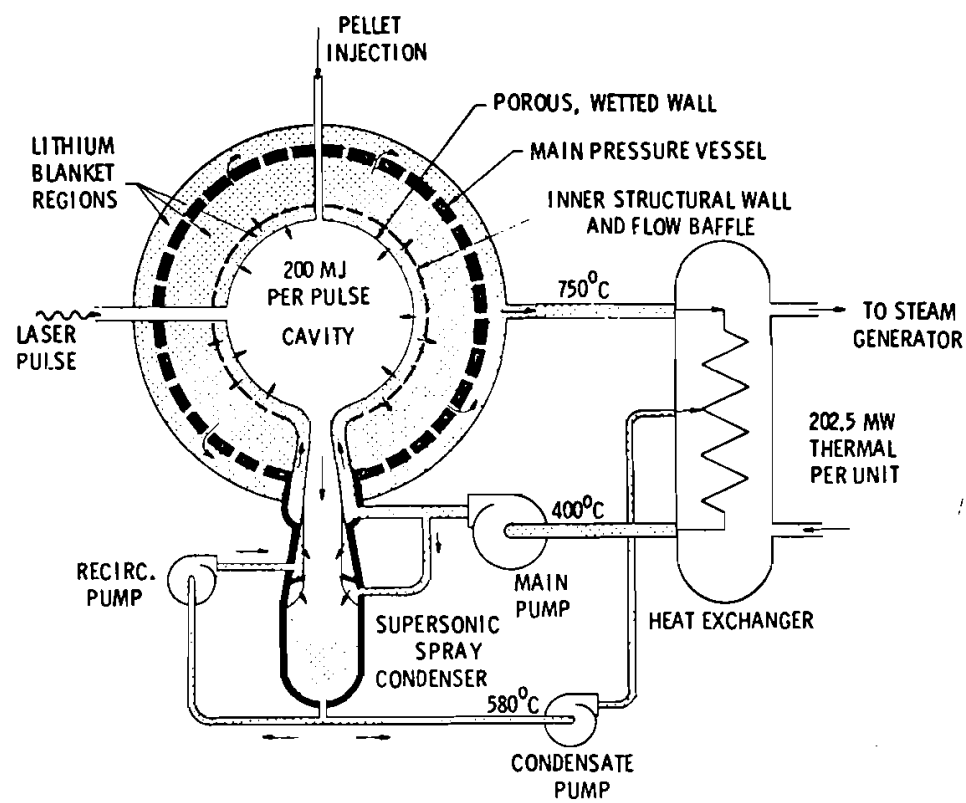

FIGURE 9 Cross Section and Flow Schematic of the Wetted Wall Laser-Pellet Fusion Power Plant

Figure 10 shows a cross section of the reactor cavity proposed in the Blascon conceptual design. In this design the reactor cavity is defined by the surface of a vortex in a swirling pool of liquid lithium. Shock waves from fusion microexplosions are partly dissipated by the cushioning effects of bubbles entrained in the liquid lithium. Remaining blast effects are resisted by a

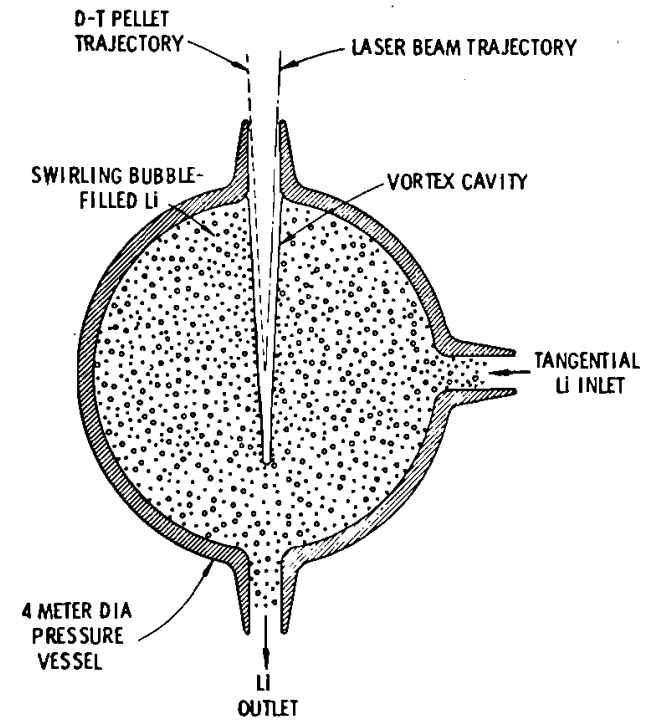

FIGURE 10 Cross Section of the Blascon Pressure Vessel and Lithium Vortex 
thick outer pressure vessel. By tailoring microexplosion energy release and vortex diameter, vaporization of the lithium is limited so that blast stresses in the outer vessel remain less than stresses due to containment of the swirling pool of lithium.

\subsection{Plasma Heating Systems}

The fuel is heated to thermonuclear temperatures in several different ways in the designs encompassed by the reference envelope. Although all system concepts function to transmit energy to the fuel, different carriers and means of excitation are employed.

Plasma Current - In Tokamaks the megampere plasma current necessary for confinement also contributes to plasma heating. The current is primarily carried by plasma electrons, which are more mobile than the fuel ions due to their smaller mass. Collisions transfer energy from electrons to fuel ions, thus the fuel heating is due to resistive interactions of the plasma current.

The heating effects of plasma current will probably not be sufficient to achieve Tokamak ignition because energy losses from the plasma increase as it gets hotter, while heating effectiveness of the current decreases. Therefore, additional heating by other methods will be required. Neutral Beams - Energetic beams of neutral fuel atoms are used to heat the plasma in Tokamak and mirror fusion power plants. These atoms will be produced by electrostatically accelerating charged fuel ions and then passing them through a gas cell where they are neutralized. The beams can then pass through the magnetic confinement field to the plasma, where they are ionized by collisions. The resulting energetic ions are trapped by the magnetic confinement field and serve to heat and fuel the plasma. A neutral beam injection system is illustrated in Figure 11. Plasma stability requires that the beam have high energy to penetrate to the plasma center. In addition, high ion currents must be produced by the accelerators to supply sufficient heating power. Consequently, plasma heating by neutral beams will require significant feedback of

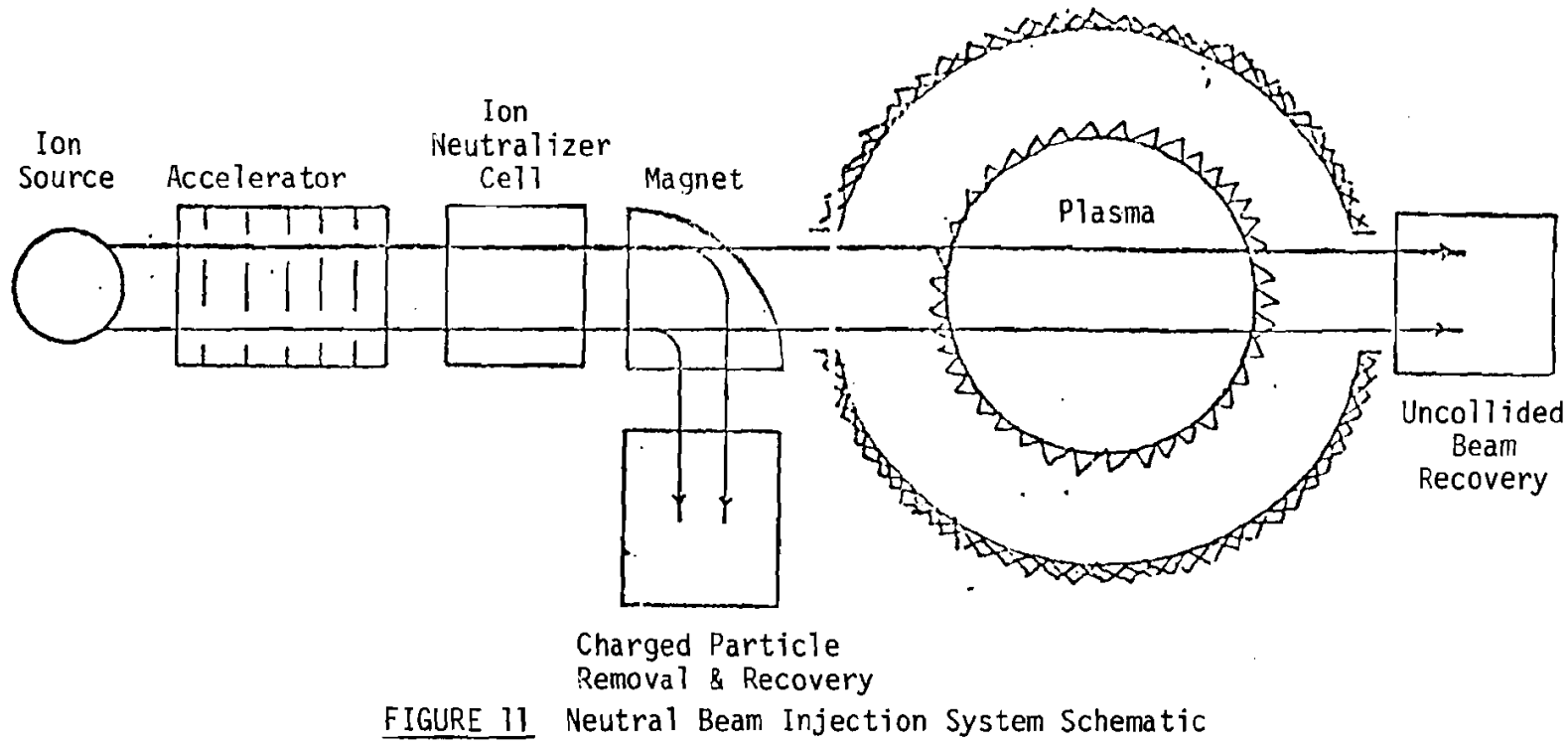


electrical power from the generating system. Therefore, net power plant efficiency will be affected by the fraction of the burn time during which plasma heating is required. Most Tokamak design concepts require neutral beam heating only during reactor startup, prior to ignition and the establishment of operating plasma conditions. However, continuous beam operation will be required in the mirror confinement concept and in Tokamak designs where neutral beams function to fuel the plasma or to control plasma conditions by feedback stabilization.

Magnetic Implosion - This method of plasma heating is used in the theta pinch conceptual design. Establishment of a strong magnetic field within a fraction of a microsecond induces a peripheral sheath of current about the plasma which is then driven inward. This imploding current sheath acts as a moving piston, imparting energy to the plasma ions. The magnet coil responsible for implosion heating is located inside the larger coil which confines the plasma and further heats it by compression. (Figure 8 )

Magnetic Compression - The energy which is supplied to a plasma during compression is distributed among the plasma particles, resulting in increased particle velocities and thus increased plasma temperature. Plasma compression is accomplished by increasing the strength of the confining magnetic field.

In the theta pinch design concept, following initial plasma heating by magnetic implosion, the confining magnetic field is increased until thermonuclear ignition occurs as a result of compression heating.

Compression heating is also possible in Tokamak devices. The toroidal magnetic field is not constant across the plasma chamber; it increases with decreasing major radius. It is possible to shrink the major radius of the plasma ring, moving it into a region of higher toroidal field and thus compressing it, by increasing the strength of the vertical magnetic field. Figure 12 shows the ATC experimental device in which this principle was demonstrated.

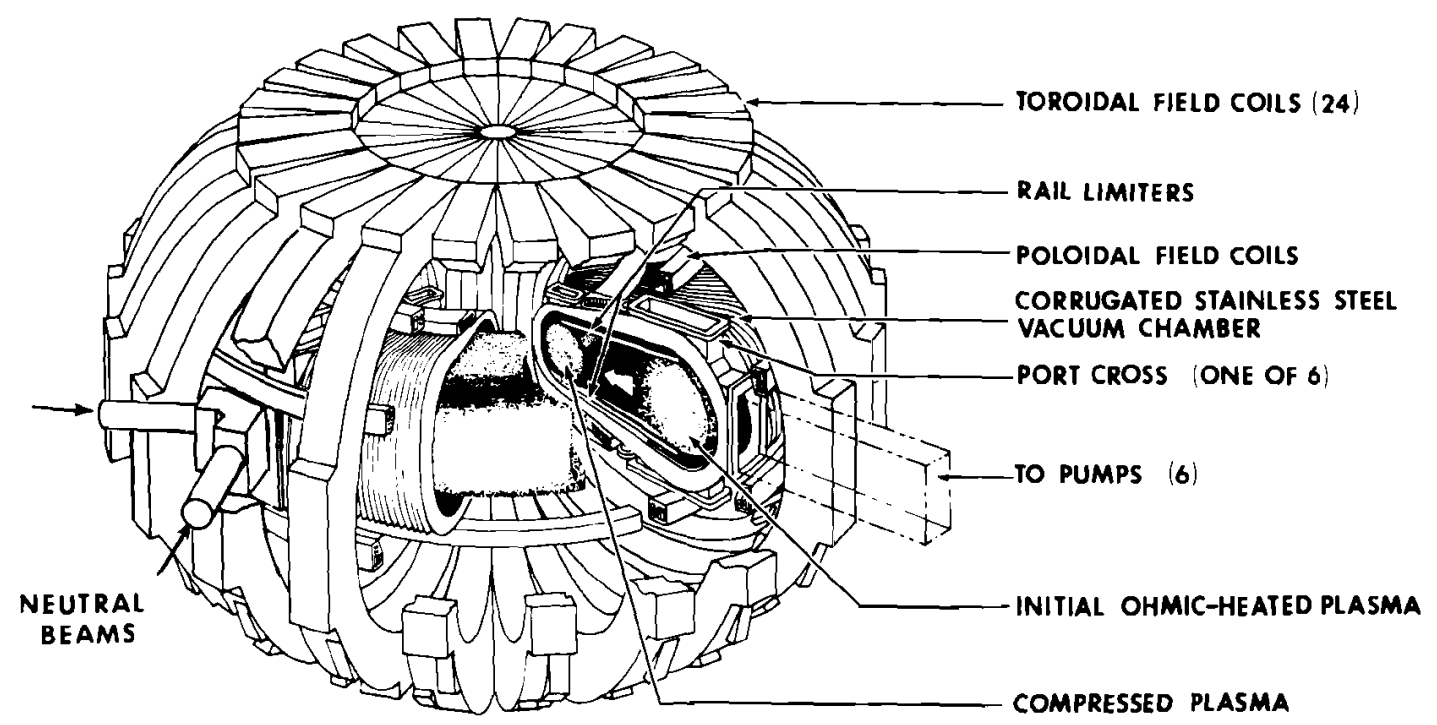

FIGURE 12 Demonstration of Compression Heating of Tokamak Plasma in the Adiabatic Toroidal Compressor (ATC) Experiment 
Compression heating is used in present mirror confinement experiments ( $2 \times$ II $B$ ) and could be employed in power plant startup.

Laser - Inertial confinement conceptual designs require that laser beams heat and compress a $D-T$ fuel pellet sufficiently that thermonuclear fusion reactions are ignited in the pellet center. In addition, pellet conditions must be achieved such that the ensuing thermonuclear burn is extensive enough to release many times the energy delivered to the pellet, before the pellet is blown apart. Beams of energetic electrons have also been proposed for pellet compression and heating.

To perform this function laser light must be simultaneously focused on the pellet from all sides, causing explosive ablation of the pellet surface. The ablation causes a shock wave in the pellet which travels inward and converges on the center of the pellet. A focusing effect is achieved by the convergence of the inward-traveling shock wave, resulting in a thousand-fold compression of material in the pellet center. This implosion shock heats the pellet core, igniting a thermonuclear burn which propagates outward. About one quarter of the pellet material undergoes fusion before the burn is quenched by pellet disassembly.

The type of laser that will be best for this function has not yet been determined. However, it is expected that a large fraction of the megajoule of energy must be delivered to each pellet by a carefully time-tailored light pulse, within a period of several nanoseconds. Furthermore, the laser must have an efficiency of about 5 percent in order to keep in-plant requirements for electricity acceptably low. The laser must also be capable of continuous operation at pulse rates of tens of pulses per second.

Uniform illumination of the pellet surface is generally expected to be achieved by directing several converging beams on the pellet simultaneously. However, in the Blascon conceptual design only one laser beam can enter the cavity through the vortex in the swirling lithium. In this case uniform surface illumination is achieved by mounting the D-T pellet at the focus of a parabolic mirror made of 1 ithium metal, as illustrated in Figure 13.

In the design concepts requiring multiple-beam illumination of the pellet the beam from a single laser oscillator will be split and used to trigger multiple laser amplifier systems. By this method several powerful beams can be simultaneously focused on the pellet from different directions.

The laser amplifier systems required to achieve desired energy outputs will be large. Several stages of amplification may be required, plus optical elements such as mirrors and plasma chamber windows. Final beam diameters of the order of a meter are anticipated. Figure 14 schematically illustrates a laser system proposed as a research tool. Although this system will yield only 0.01 megajoule pulses, it dwarfs the human figures shown in the foreground. 


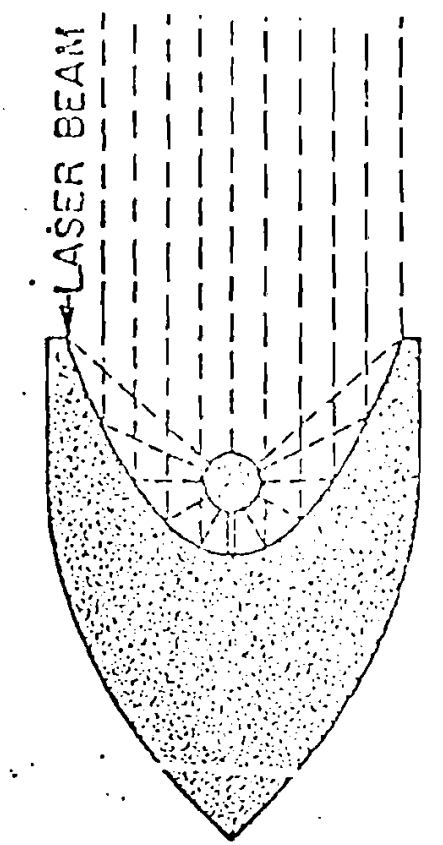

FIGURE 13 Section Through a D-T Pellet Mounted at the Focus of a Parabolic Mirror

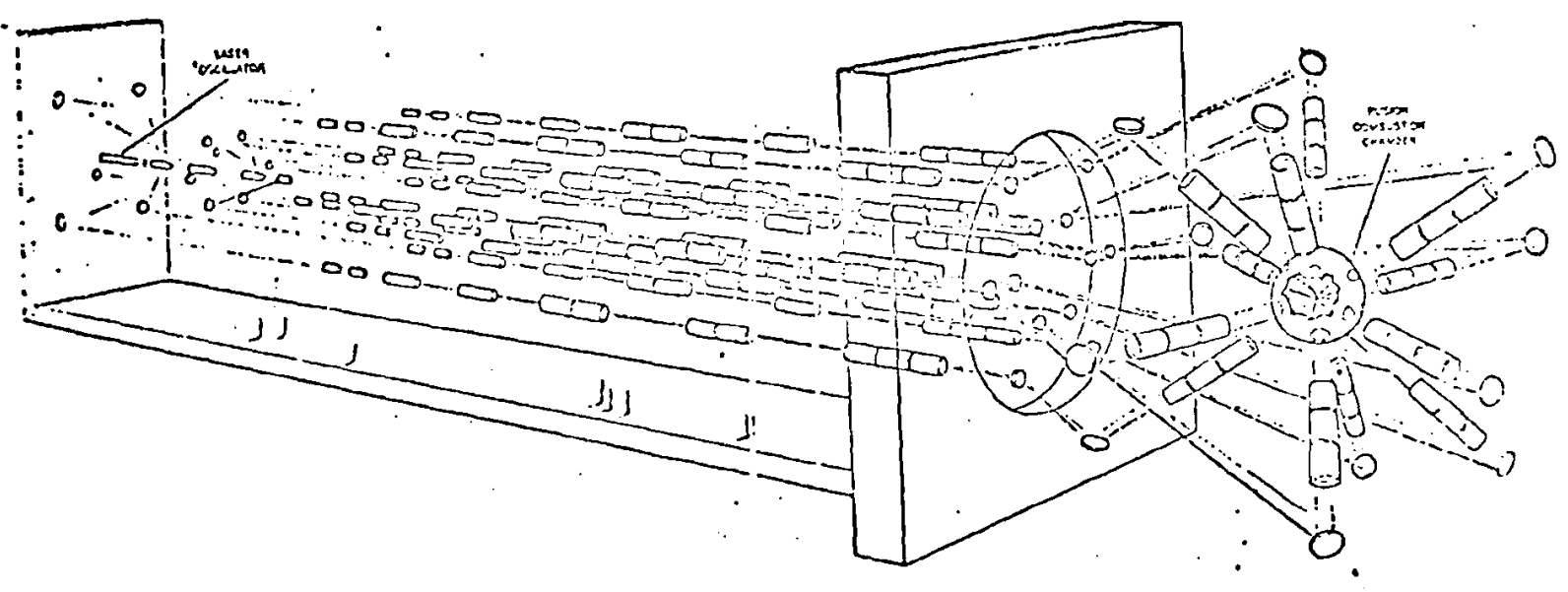

FIGURE 14 Schematic Drawing of a Planned Experimental Laser and Pellet Irradiation Facility

\subsection{Fuel Systems}

Although the energy release per $D-T$ fusion is a constant, the fueling requirements for the various conceptual designs vary considerably. This is due to differing amounts of neutron energy multiplication in the blanket, differing thermal efficiencies, differing in-plant requirements for the recycle of electrical power, and differing burnup fractions of supplied fuel. 
Fuel systems purify the D-T fue1, prepare it in the form necessary for use, and inject the appropriate quantity into the reaction chamber at the appropriate time and speed. The systems differ mainly in the method of injection, which is primarily determined by the confinement method and the duration of the thermonuclear burn.

Purification - Nuclear reactions in the plasma produce, in addition to helium ash, significant amounts of protium $\left({ }^{1} \mathrm{H}\right)$ which must be removed from the D-T $\left({ }^{2} \mathrm{H}-{ }^{3} \mathrm{H}\right)$ fuel before the fuel can be recycled to the plasma. In the two conceptual designs which treat this step in detail, cryogenic distillation is used for separation of the hydrogen isotopes. An initial precooler traps out any contaminants less volatile than hydrogen and helium, as it cools the fuel to its dew point for introduction to the primary distillation column. Distillation yields a vapor fraction containing $\mathrm{He}, \mathrm{H}_{2}$, and some $\mathrm{H}-\mathrm{D}$ and $\mathrm{H}-\mathrm{T}$ contamination. This vapor is sent to a secondary distillation column where it is redistilled to remove tritium contamination. The liquid fraction produced by the primary distillation column, containing $T_{2}, D-T, D_{2}$, and sufficiently small contamination of $H-T$ and $H-D$, is retained for recycle as fuel.

Preparation - The fuel injectors require either gas or solid pellet feed. The liquid fuel stream resulting from distillation is either warmed and vaporized or further cooled to solid temperatures by liquid helium. Pellet production may be achieved by extrusion, cutting and shaping of solid D-T material; by the cooling of droplets formed by the breakup of a liquid jet; or by enclosing fuel gas in minute glass bubbles.

Injection - Systems have been designed to inject fuel in the forms of a gas, beams of neutral atoms, and pellets. Tokamak and theta pinch reactors begin their cycles with neutral fuel gas in their chambers. In addition, the theta pinch reactor requires injection of neutral gas between the plasma and the chamber wall during the thermonuclear burn. Gas will be injected by straightforward valving and metering systems.

Plasma replenishment by neutral atom beams is used in Tokamak and mirror devices in spite of the large amounts of electrical power that it requires. The operation of the injectors has been described in the section on plasma heating, and is indicated schematically in Figure 11. Fuel is supplied to the neutral beam injectors as a gas for subsequent ionization, acceleration, neutralization and injection.

The Tokamak and laser-pellet reactors are fueled by pellet injection. Millimeter-sized pellets will probably be employed in both cases to achieve deep penetration of Tokamak plasma prior to pellet evaporation and to provide an appropriate amount of fuel for each laser-pellet microexplosion.

The method of pellet injection into Tokamak devices is as yet unspecified. Pellet velocities near $10^{5}$ meters per second appear to be necessary to achieve adequate plasma penetration prior to pellet evaporation. Electrostatic acceleration of pellets to such velocities is expected to require more than $100 \mathrm{million}$ volts, which is beyond the capabilities of present systems. If pellet evaporation rates have been overpredicted, so that pellets of 0.01 millimeter diameter can be used at the same velocities, existing accelerator technology may suffice. 
Pneumatic or mechanical injection of pellets at velocities near 100 meters per second is projected for laser-pellet designs. Pneumatic pellet injection into Tokamaks is also possible, al though the effects of the driver gas on the plasma are not known. A conceptual device using liquid helium to freeze D-T fuel for extrusion, followed by laser-bean cutting and shaping and pneumatic injection is shown in Figure 15. A rotating valve arrangement which operates synchronously with pellet injection to protect the injector from the effects of fusion microexplosions is also illustrated.

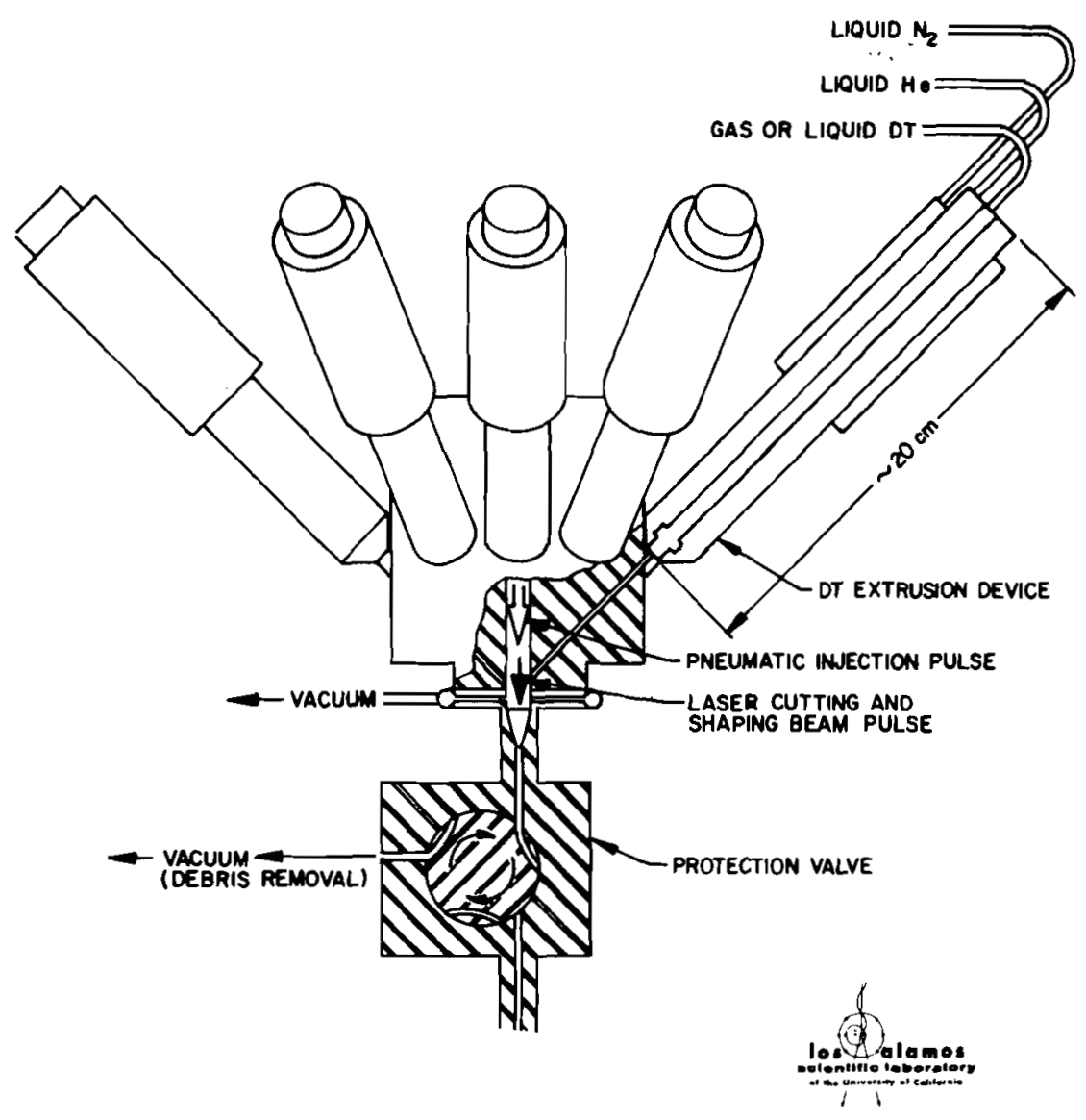

FIGURE 15 Conceptual Fuel Pellet Fabrication and Injection System

\subsection{Blankets}

Functions - The blanket of a fusion reactor functions to:

- Convert neutron energy to heat,

- Breed tritium fuel by neutron interactions with lithium,

- Contain the fuel and other particles escaping from the plasma,

- Provide a vacuum chamber for the plasma (in some cases), 
- Shield outer structures from fusion neutrons (which it moderates and captures) and other energy sources,

- Contain the tritium bred within it, and

- Contain the coolant which transports heat from the blanket structures.

Materials - The functions performed by the blanket place a variety of conflicting requirements upon blanket materials. These materials must be able to withstand high temperatures and the damaging effects of fast neutrons and be chemically compatible. They must be efficient moderators of fast neutrons, and neutronically compatible with the breeding of tritium. The materials must be of a type that can be procured and fabricated.

All of the proposed blanket designs include lithium for the breeding of tritium. However, inclusion as a liquid metal, a molten salt, or a solid compound depends upon the lithium's additional function as a coolant, a neutron moderator, or a neutron multiplier.

- Coolants

Liquid lithium metal and helium gas are proposed as blanket coolants. When liquid lithium is used, blanket design is simplified because the lithium functions simultaneously as a coolant, moderator and fertile material. However, several problems are encountered. Considerable pumping losses occur when conducting materials are pumped across magnetic fields. High temperature liquid lithium is corrosive to stainless steel and nickel alloys and significant residual inventories of bred tritium are expected due to the solubility of tritium in liquid lithium. Use of helium coolant requires that the blanket become a heat exchanger. Additional structure will be required in the form of tubing and flow channels. However, corrosion problems will be reduced and higher temperature operation may be achieved. Low tritium inventories are expected to be maintainable in the helium coolant.

- Structural Materials

Stainless and nickel steels, refractory niobium-zirconium (Nb-Zr) alloy, and aluminum alloys are proposed as blanket structural materials. The present metals industry can supply large quantities of stainless steel of consistent quality. The excellent high temperature properties of refractory metal alloys promise higher blanket temperatures and improved thermal efficiencies, but will require the development of an industry capable of supplying and fabricating these materials in large quantities. Blanket structures made of aluminum alloys may have less long-lived radioactivity induced by neutron interactions than steel or niobium structures but must be limited to low temperatures to retain adequate tensile strength.

- Neutron Multiplier

Blanket designs which restrict the amount of ${ }^{7} \mathrm{Li}$ in the blanket, or which employ materials which can compete successfully with ${ }^{6} \mathrm{Li}$ to capture neutrons, require the inclusion of a neutron multiplying material in the blanket. The tendency of a beryllium nucleus to emit two neutrons when struck by a single neutron of high energy is sometimes used to increase the number of neutrons available for tritium production by ${ }^{6} \mathrm{Li}$. 
- Neutron Moderator

Carbon and beryllium are included in several designs for their neutron moderating properties. Some designs include significant extra amounts of stainless steel structural material because inelastic scattering and neutron production reactions in iron efficiently moderate high energy neutrons.

- Shield

In designs using superconducting magnets, an outer blanket layer called a shield is included to further attenuate fluxes of particles, radiation and heat. Lead, boron carbide, stainless steel, hydrocarbons and concrete are used.

Primary Combinations - The following combinations of materials are used in the blankets: 1) 1iquid lithium moderator, fertile material and coolant, in stainless steel or niobium structures; 2) liquid flibe moderator and fertile material, with helium gas coolant, in nickel alloy structure; 3) solid lithium alloy fertile material, with helium gas coolant, in stainless steel or aluminum alloy structure.

First wall - The plasma chamber is bounded by the inner wall of the blanket. The inner wall receives most of the energy released in the fusion process which must be transmitted into the interior of the blanket, for removal by the coolant. The first wall must also support internal blanket pressures and prevent leakage of fluid blanket contents into the plasma chamber. The difficulties of satisfying both the requirements are compounded by radiation-induced erosion and transmutation effects.

A variety of first wall designs is used. In some designs the first wall is protected from the direct effects of particles and radiations emanating from the plasma. Magnetic divertors or direct conversion generating systems collect charged particles. Inner shields of carbon cloth or coolant-carrying tube banks intercept particles and radiation. Ablative coatings of liquid lithium function similarly in the laser-pellet designs. In the theta pinch design the first wall is coated by a thin layer of refractory insulator material to electrically insulate the plasma from the wall.

In the toroidal designs that employ liquid lithium coolant, the first wall is composed of the curved surfaces of the blanket segments, as sketched in Figure 16. The pressure forces on the first wall are supported by tensile stresses in the wall and segment structure. In the mirror design the first wall is formed by the surfaces of the innermost tubes which view the plasma since the blanket is composed of a shell of tubes carrying the lithium coolant. Pressure forces are supported by hoop stresses in the structure.

The first wall concepts of the lithium cooled laser-pellet designs are discussed under inertial confinement.

The helium cooled Tokamak design employing a liquid flibe moderator is similar to those with liquid lithium coolant in that the blanket is segmented but the blanket first wall is not ribbed. It is formed from a smooth plate honeycombed with rectangular coolant flow channels. This is 
shown in the cross sectional view of Figure 17 which also shows the radiation shield layer of coolant carrying tubes interposed between the wall and the plasma. The flibe pressure forces on this wall are carried by tensile stresses in the support plates which segment the blanket.

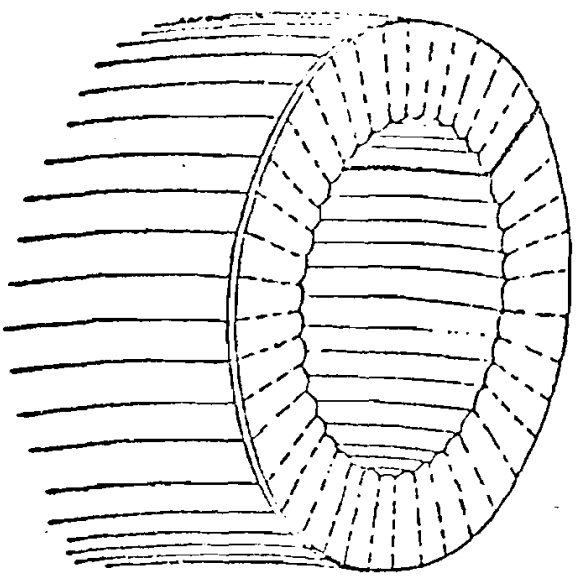

FIGURE 16 Section Through a Segmented Blanket Showing Ribbed First Wall Stressed in Tension

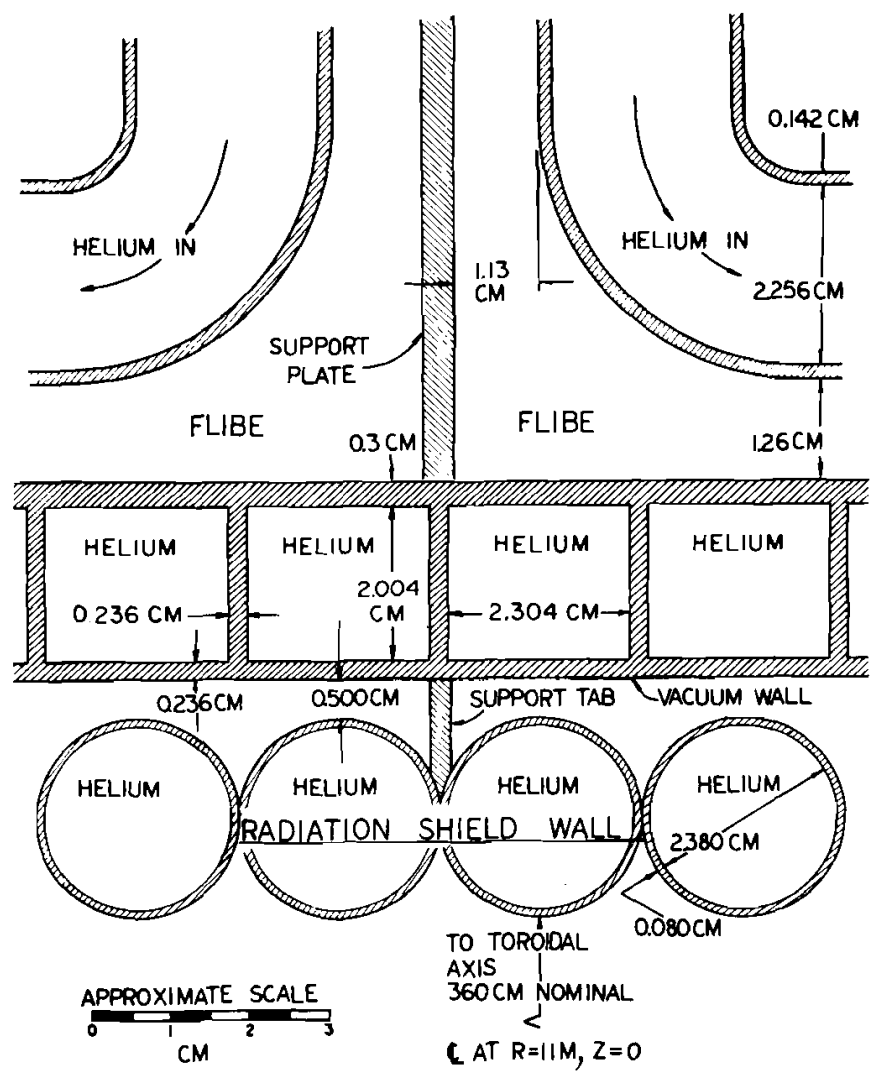

FIGURE 17 Sectional View of the First Wall and Radiation Shield of a Helium Cooled Blanket Containing Liquid Flibe 
Two Tokamak blanket designs use solid compounds of lithium and helium cooling. In both designs the first wall must support coolant forces. The UWMAK-II design employs a ribbed inner wall conceptually similar to the Tithium cooled Tokamak designs. Flow channels for helium coolant are incorporated in the first wall ribbing, and woven carbon cloth provides an inner shield.

In the Brookhaven National Laboratory (BNL) blanket design segmentation is extended, and the blanket is composed of cylindrical canisters whose domed ends comprise the first wall as illustrated in Figure 18. The blanket pressure forces are supported by tensile stresses. Because the aluminum alloy structure must be maintained at low temperatures compared to those desired for thermodynamic efficiency, the aluminum canisters are water cooled while the contents of the canisters are helium cooled. The canister shells contain interior coolant channels constructed by bonding two layers together. Parallel channels travel down one side of the canister across the domed canister end and back up the other side.

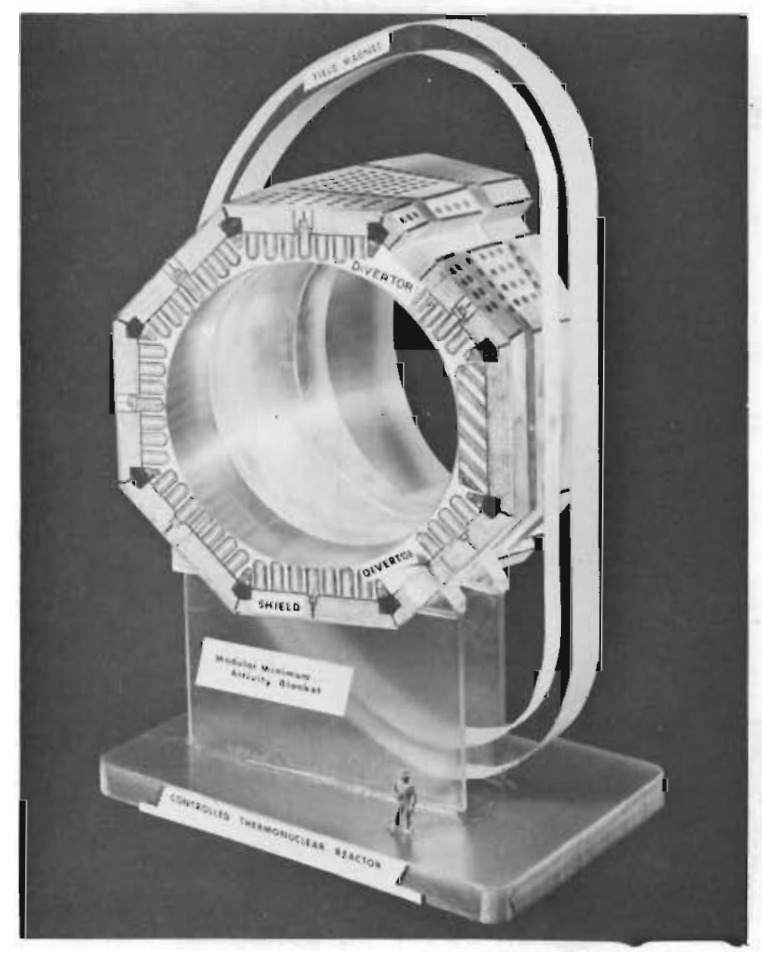

FIGURE 18 Blanket Composed of Cylindrical Canisters Whose Domed Ends Form the First wall

Blanket Interior - The materials in the blanket interior moderate the fusion neutrons, provide flow channels for the coolant which removes heat, and absorb the slowed neutrons, breeding tritium in the process.

In designs employing il thium as a coolant, the blanket is composed primarily of lithium and structural materials to support and channel it. However, in some cases, to adequately attenuate 
the high energy neutrons with a reasonable blanket thickness, other neutron moderating materials are also employed. Graphite and stainless steel are variously specified for this function. In general, the first wall is followed by a lithium layer for tritium production via the ${ }^{7} \mathrm{Li}$ reaction, then by a moderating layer, and then by a final lithium layer to absorb the slowed neutrons and breed tritium via the ${ }^{6} \mathrm{Li}$ reaction. These general comments also apply to the tube-bank blanket of the mirror design; graphite is included in outer blanket regions within the tube blankets.

Where minimizing blanket thickness is desirable, as in the theta pinch design, the moderator layer is thickened at the expense of the initial lithium layer. However, the concomitant reduction in ${ }^{7} \mathrm{Li}$ reactions reduces tritium breeding, and may require an additional inner layer of neutron multiplying material to maintain an adequate breeding ratio. In the region following the moderator, tritium breeding via ${ }^{7} \mathrm{Li}$ reactions is reduced due to attenuation of the flux of fast neutrons. Consequently, a further reduction in blanket thickness can be achieved by removing ${ }^{7} L i$ from this region and using 1 ithium enriched in ${ }^{6} \mathrm{Li}$.

In the helium cooled blanket employing molten flibe (a eutectic mixture of salts of LiF and BeF 2 ) as moderator and fertile material, the blanket is primarily composed of flibe, plus structural material to support and channel the flibe and the helium coolant. The beryllium present in the flibe produces enough additional neutrons through multiplication reactions to make up for parasitic neutron captures in the fluorine.

In the helium cooled blankets which contain solid lithium compounds, a beryllium region is located close to the first wall to achieve multiplication of fast neutrons. The beryllium is preceded or intermixed with lithium compounds to absorb low energy neutrons emanating from the beryllium. The neutron multiplying region is followed by regions containing lithium compound and graphite, to moderate and absorb energetic neutrons penetrating the beryllium region. The difference between the two proposed designs is that in the UWMAK-II design the blanket contents are canned in stainless steel and isolated from the helium coolant, while in the BNL design the helium flows through the blanket particles as through a pebble bed.

Blanket cooling - The heat energy deposited in blanket materials is removed by circulating the primary coolant through the blanket. The rate of energy deposition is greatest in first wall structural materials, and decreases rapidly with distance from the first wall as the energycarrying neutrons are slowed and absorbed. Consequently, coolant is commonly circulated to the first wall vicinity and then through regions of lower power density located deeper in the blanket. This flow pattern assists in maintaining the first wall temperatures below the maximum permissible.

In the magnetic confinement designs which use liquid lithium coolant, circulation patterns are influenced by the need to minimize the power required to pump the coolant across magnetic field lines. In the Oak Ridge National Laboratory (ORNL) design the lithium flow is directed in the toroidal direction, parallel to the direction of the confining magnetic field. This may be seen in Figure 19 which illustrates the flow in one of the ribbed blanket sectors that was sketched in Figure 16. In blanket designs which use liquid lithium coolant, considerable heat is 


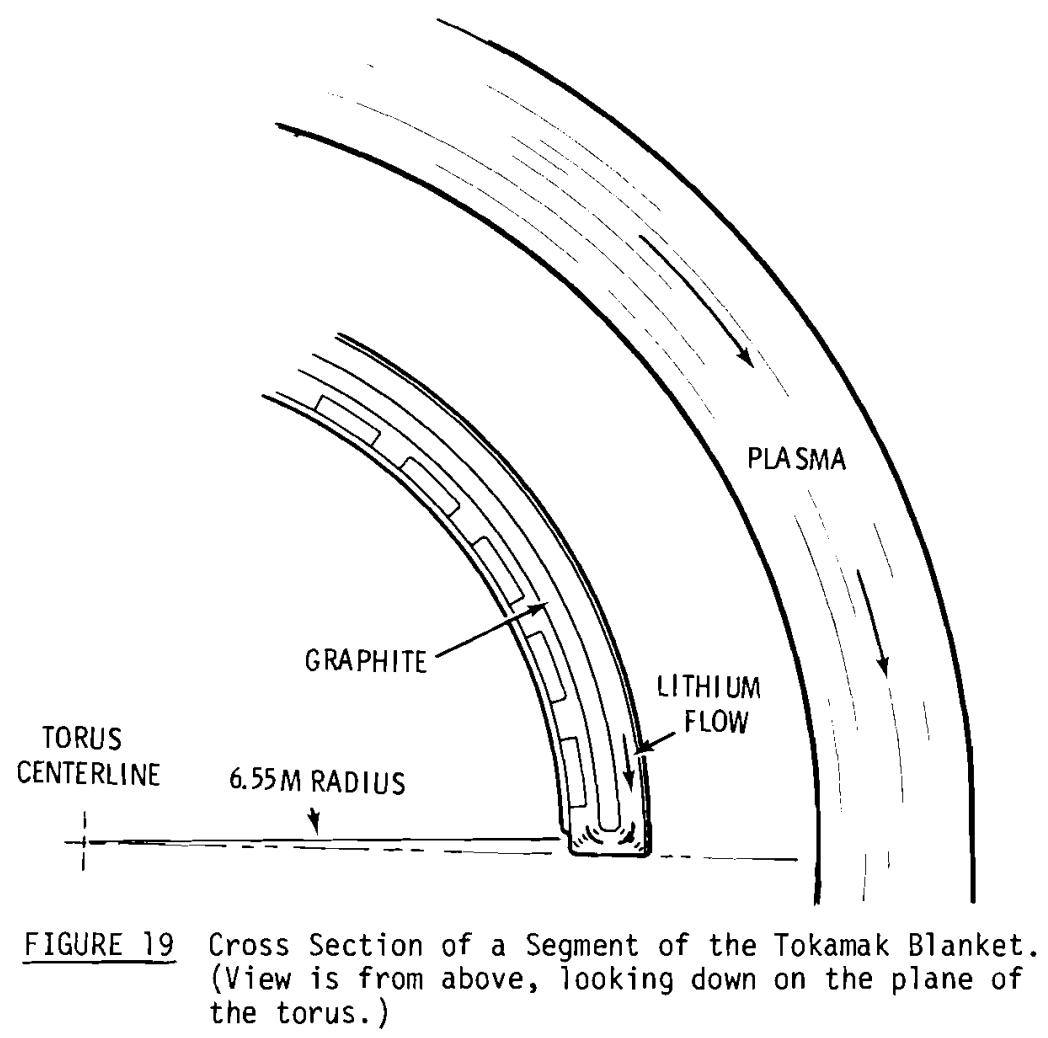

deposited directly in the lithium itself. Because the lithium flow tends to follow magnetic field lines, mixing is inhibited. Due to the strong radial dependence of power deposition, nonuniform coolant temperatures become possible. Consequently, in the UWMAK-I design the coolant is forced to make a series of radial traverses while it flows around the blanket periphery. This flow pattern is indicated in Figure 20. In this case pumping losses are minimized by directing the peripheral flow perpendicular to the toroidal magnetic field.

In the mirror design the lithium carrying tubes which form the blanket are directed along the lines of the magnetic field which confines the plasma. This may be seen by comparing the blanket illustration of Figure 21 with the field lines shown in Figure 6.

In the theta pinch design the magnetic field halts the flow of the lithium coolant when it is pulsed on for the thermonuclear burn. Coolant flow takes place only after the burn is completed, lasting until initiation of the next burn cycle. Because this coolant flow occurs in the absence of a magnetic field the sequence of radial and toroidal flows indicated in Figure 22 does not impose the power-loss penalty.

In the ablative wall laser-pellet designs lithium flow is unimpeded by magnetic fields, and is primarily radially outward from the first wall to outer regions (Figure 9). The blascon blanket, which lacks a structural first wall, is an exception to the rule. In the blascon blanket the lithium swirls inward toward the central vortex from the tangential inlet port. It is then drawn to the central outlet port in the chamber bottom (Figure 10). 


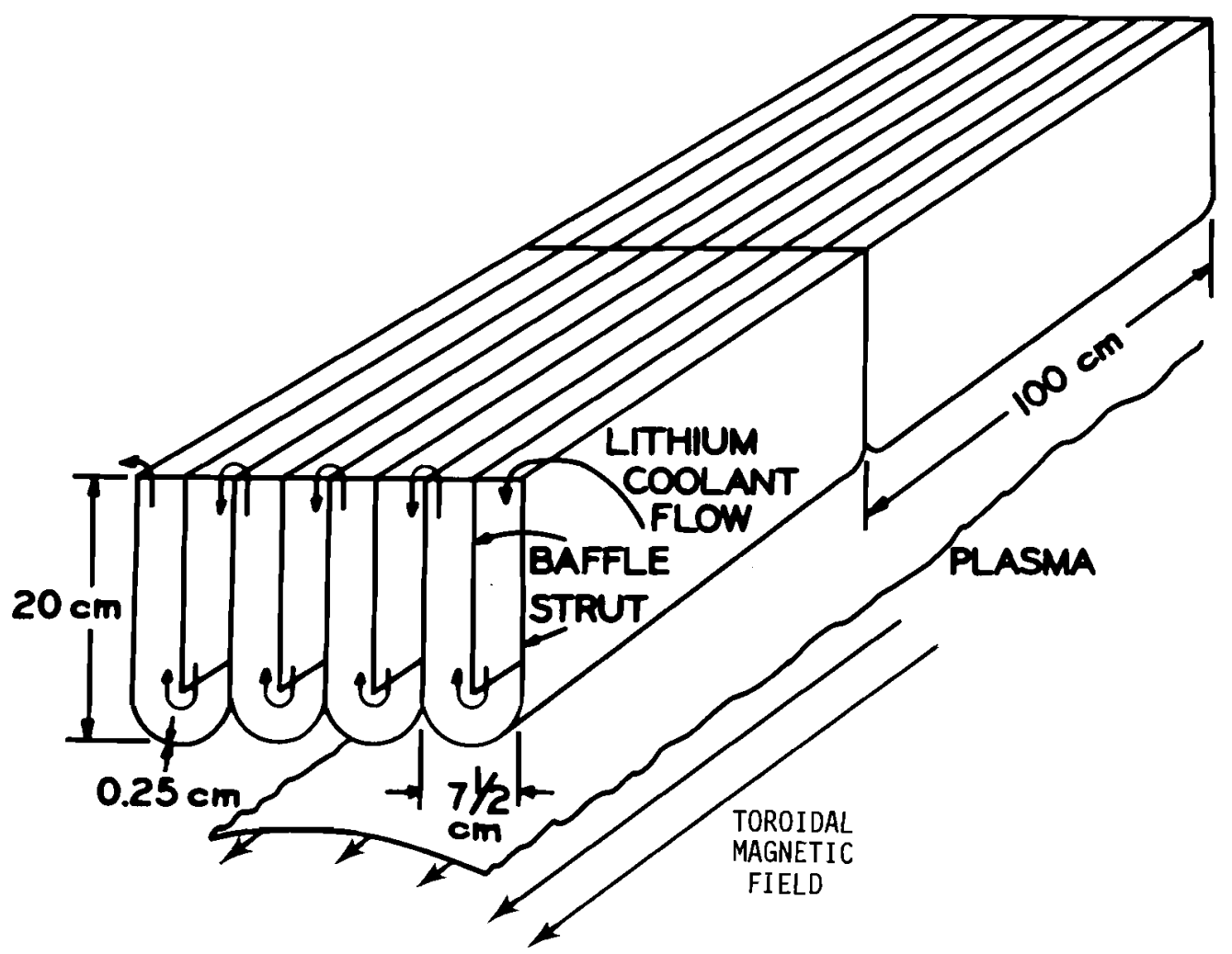

FIGURE 20 Flow Pattern of Lithium Coolant Near the First Wall of the UWMAK-I Design

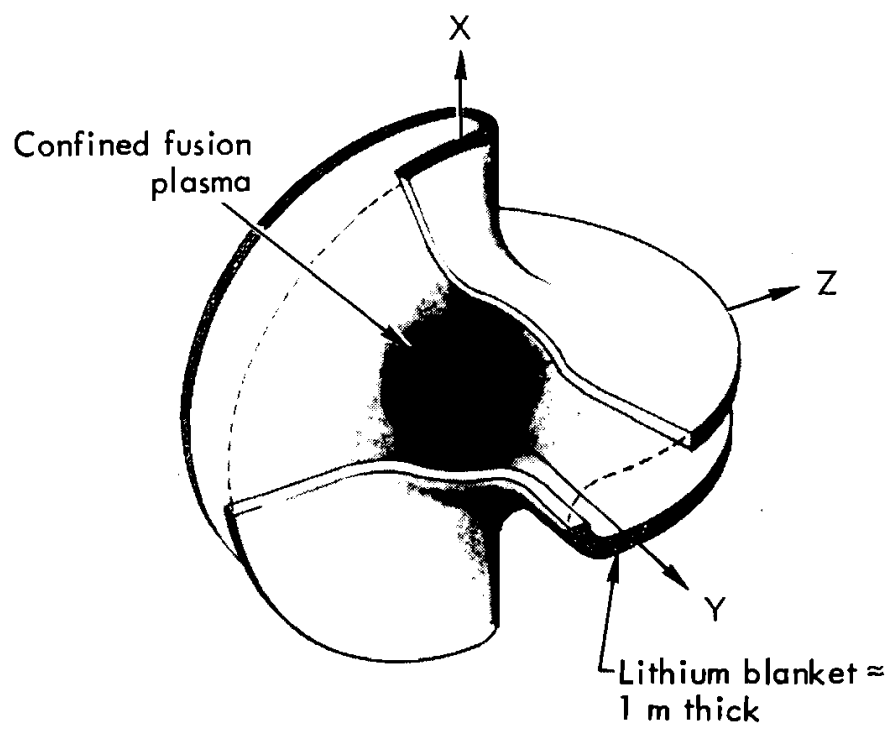

FIGURE 21 Blanket of Tubes Carrying Lithium Coolant Along Magnetic Field Lines of the Mirror Confinement Design 


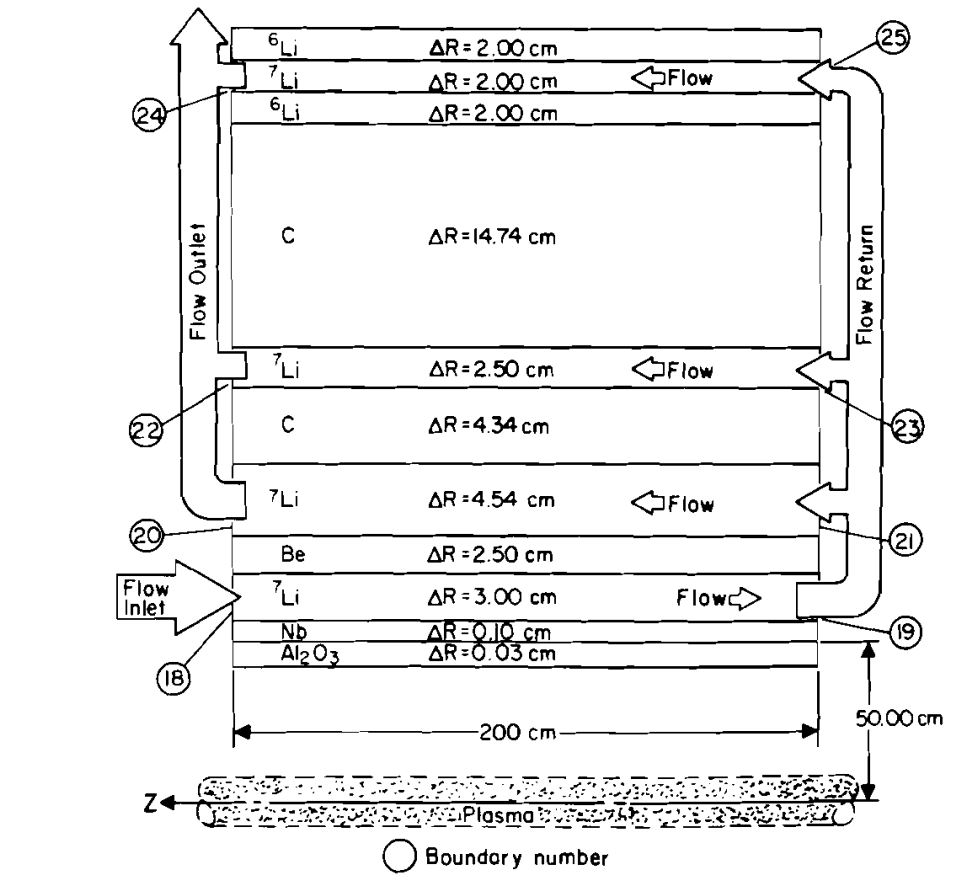

FIGURE 22 Schematic Blanket Section Showing Material Layout and Coolant Flows for the Theta Pinch Design

Although considerable heat energy is deposited directly in lithium coolants, this is not so for helium coolants. High pressure helium must be ducted throughout the blanket in helium cooled designs. Thus, the molten flibe blanket of the Princeton Plasma Physics Laboratory (PPPL) Tokamak design is honeycombed with helium flow tubes, as shown in Figure 23 . The flibe is circulated for tritium and impurity removal, not as the primary coolant.

In the helium cooled UWMAK-II blanket the helium flows first from headers located in the outer blanket to the inner wall, and then back over the blanket contents to outlet headers (Figure 24). In this case the helium is not ducted in tubes but flows over the surfaces of stainless steel pins containing moderator and fertile materials.

In the helium cooled BNL blanket design, the helium flows into each of the individual blanket modules down an annular space immediately inside the water-cooled outer wa11. After reaching the domed end of the module the helium flows through a distributor plate and then through the module contents, as shown in Figure 25. In this design the blanket materials are not canned. Consequently, bred tritium is carried away by the coolant flow.

Shields - Superconducting magnet coils are specified in all of the magnetic confinement designs but the theta pinch design. Because neutron and gamma radiation penetrating the blanket can cause degradation of superconductor properties and increase the heat load upon the cryogenic refrigeration system, shields are incorporated between the high temperature portion of the blanket and the superconductors. Such shields act as thermal insulation, in addition to attenuating radiation fluxes and providing for the disposition of associated heat energy. 


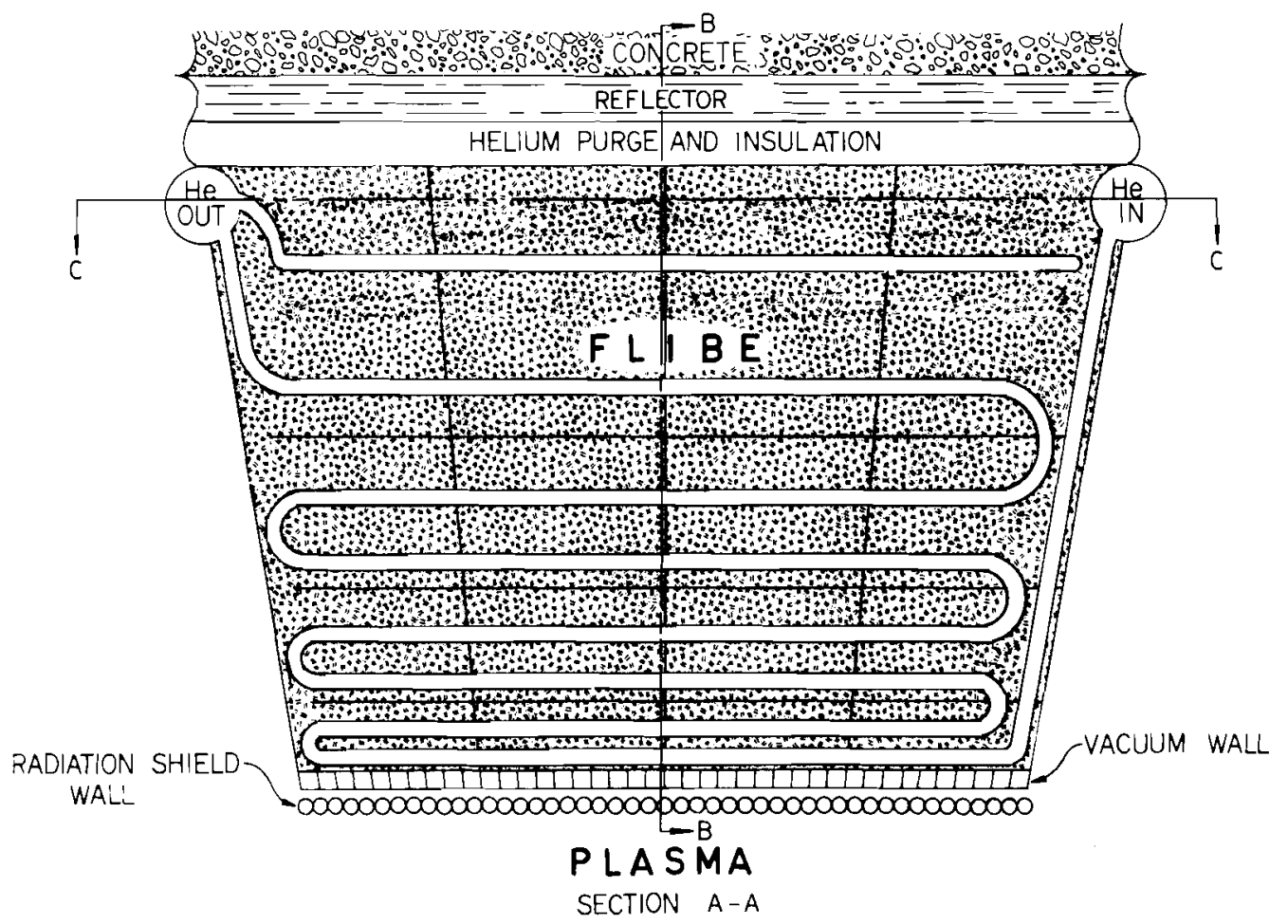

FIGLIRE 23 Helium Flow Tubes for Cooling the Liquid Flibe Blanket of the PPPL Tokamak Design

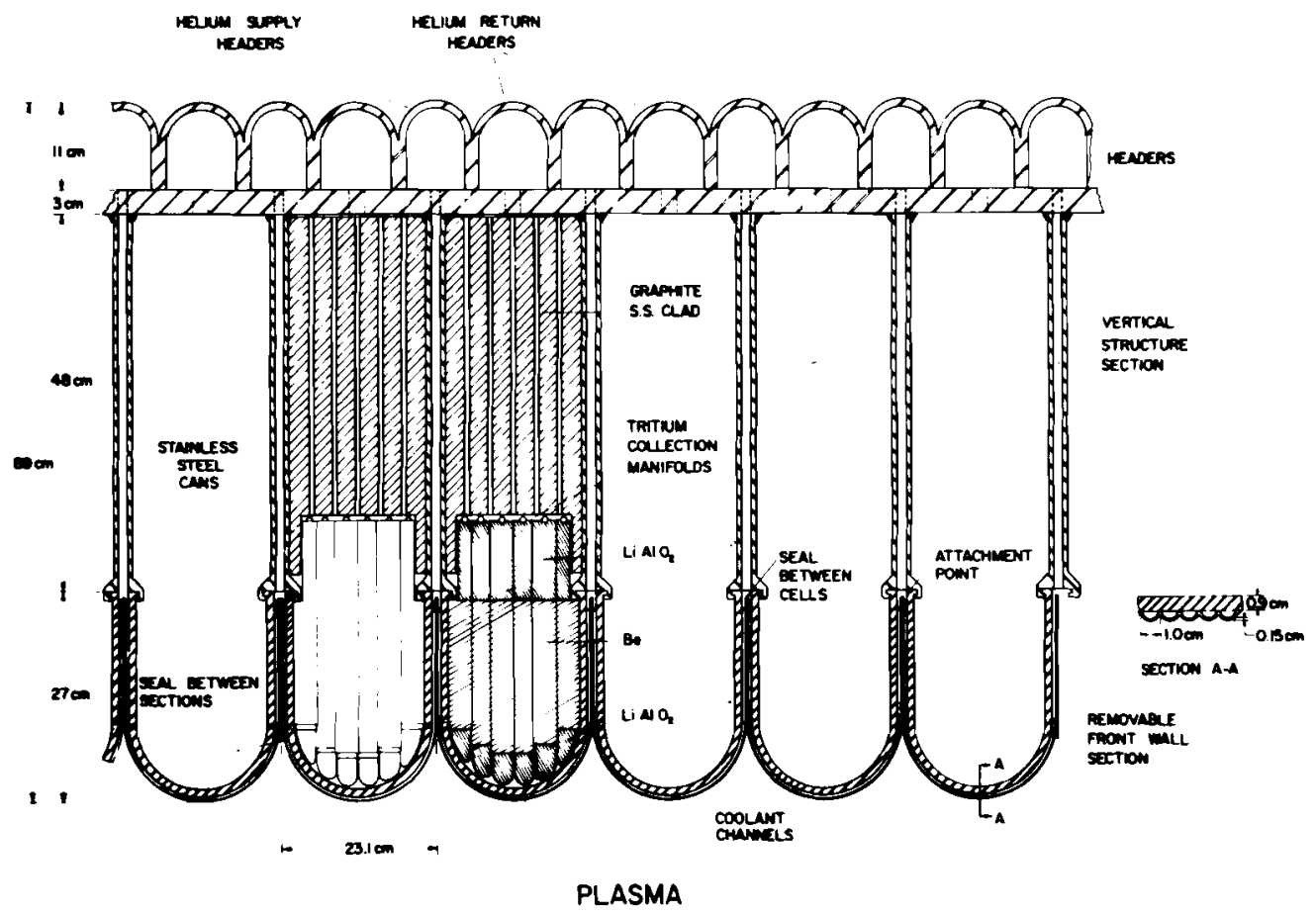

FIGURE 24 Blanket Section for the UWMAK-II Tokamak Design 


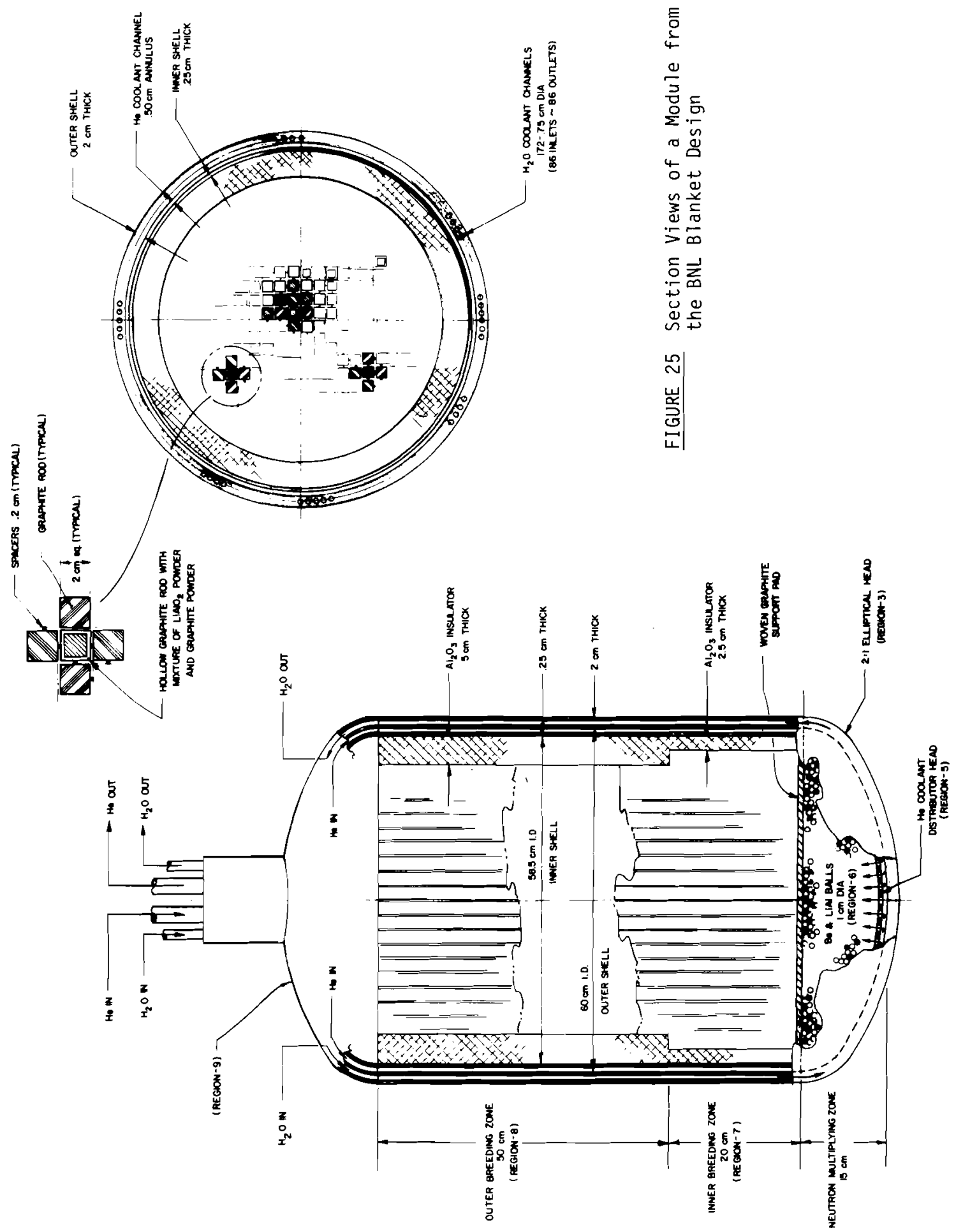


Shields are about a meter thick. They contain heavy metals (lead) for gamma shielding, and neutron moderators and absorbers (hydrocarbons, $B_{4} C$ ). During operation helium gas coolant is circulated through the shields, holding temperatures intermediate between room temperature and blanket operating temperatures.

\subsection{Heat Transfer Systems}

The heat transfer systems remove heat from blanket materials and transport it to the electricity generating systems. They contain and circulate the coolants and confine any radioactive materials that are entrained therein. Liquid lithium metal and helium gas are used as primary coolants. An intermediate coolant system is provided in designs which utilize liquid lithium primary coolant, to isolate the steam system from radioactivity in the primary coolant in case of heat exchanger leakage. Intermediate coolants include molten sodium, molten sodiumpotassium ( $\mathrm{Na}-\mathrm{K}$ ) alloy, helium gas and potassium operating in a liquid/gas binary cycle. In all systems heat is ultimately transferred to a steam system. Especially high thermal efficiency is achieved in the design with potassium intermediate coolant, by using the potassium vapor to drive a topping turbine before it is condensed in the steam generator.

Lithium Primary Coolant Systems - In lithium cooled blankets considerable heat is deposited directly in the lithium coolant. The blanket, especially its inner regions, consists primarily of structure to support and channel the lithium coolant. Additional blanket material to enhance neutron moderation is commonly located farther from the plasma, and heat transfer from it to the coolant is provided by flow channels through and around the moderator.

Maximum lithium temperatures are limited in stainless steel structures to minimize corrosion and erosion. Higher lithium temperatures are allowable in refractory metal structures although steam system temperature limitations may limit their usefulness unless a topping cycle is used.

The transfer of large quantities of heat requires the circulation of large amounts of coolant. In designs where the lithium is pumped from the blanket to an external heat exchanger, the necessary pumping head is produced by free-surface centrifugal pumps of the type developed in the LMFBR program.

In some designs the lithium coolant is not pumped from the blanket, to minimize pumping power losses caused by magnetohydrodynamic (MHD) effects. (Currents are induced in conducting fluids moving across a magnetic field; the currents interact with the field to cause a retarding force on the flowing coolant.) In the ORNL Tokamak and in the mirror designs the lithium primary coolant is circulated to heat exchangers located within the blanket. The need for locating conventional pumps in this irradiated environment is avoided by using MHD effects to pump the coolant. In these designs the forces moving the coolant are caused by passing a current through the conducting liquid metal coolant within the magnetic field of the device. However, the conceptual simplicity of MHD pumping is offset by the need for insulating flow channels for the liquid lithium at high temperatures. 
In designs where the lithium is pumped from the blanket, heat exchange is to liquid metal or alloy intermediate coolants. Shell and tube intermediate heat exchangers (IHXs) typical of LMFBR technology are used, with the lithium primary coolant on the shell side. In the designs where the IHXs are located within the blanket, the heat is removed by gas-phase intermediate coolants. (Since they are nonconducting they can be pumped from the blanket across magnetic field lines without MHD losses.) In the mirror design heat is exchanged to helium intermediate coolant. The lithium-carrying tubes which make up the blanket are manifolded together and the lithium flow is collected and led over heat exchange surfaces; the lithium is then returned to other blanket tubes via outlet manifolds. In the ORNL Tokamak design, banks of heat exchanger - tubes are located in the large ducts through which lithium flows in the outer blanket region. Potassium intermediate coolant enters the tube banks as a liquid, is boiled by heat from the lithium, and exits as a vapor.

Helium Primary Coolant Systems - Helium is not corrosive at high temperatures, al though impurities may strongly enhance corrosion. Helium does not dissolve corrosion products and constituents of structural materials and is not subject to MHD losses when pumped across magnetic fields. And in addition, the technology of helium heat transport systems has been developed and is commercially employed in High Temperature Gas Cooled (fission) Reactors. These factors offset the increased blanket complexity required when helium is used as the primary coolant instead of liquid lithium.

Helium coolant must be ducted to all parts of the blanket to collect heat deposited in structural, moderator and fertile materials. Circulation is achieved by circulators typical of existing HTGR technology.

The operating temperatures of helium cooled blanket structures made of stainless steel or nickel alloy are 1 imited by structural properties of the metals, rather than by their corrosion or erosion. Consequently, use of helium as primary coolant allows blanket operation at somewhat higher blanket temperatures and the achievement of better thermal efficiencies than are achievable in blankets cooled with lithium.

In the BNL blanket design high helium outlet temperatures are achieved despite the necessity of maintaining the aluminum structural material at much lower temperatures. This is achieved at the expense of considerable blanket complexity: the structure is cooled by a separate water cooling system; only inlet helium flow contacts the structure; and a ceramic sleeve is required in each module to thermally insulate the structure and inlet helium flow from the hotter blanket materials in the module interior. The temperature difference between hot and cold legs of the primary coolant system in this design is the largest of the various designs.

Intermediate Coolant Systems - An intermediate coolant system (ICS) separates the primary coolant system from the steam system in each of the designs employing liquid lithium as primary coolant. In addition to transporting heat from the lithium to the steam system, an ICS functions to physically separate the primary coolant system from the steam system. The primary coolant system is therefore protected from the effects of a lithium/water reaction in the event 
of steam generator leakage - both shock waves and corrosive reaction products are confined by the ICS. Because most radioactive materials in the lithium primary coolant are excluded from the steam generator, its surveillance, maintenance and repair are facilitated, and the release and dispersal of those radioactive materials by the molten metal/water reaction when leaks occur are reduced significantly.

Because helium is inert and cannot react vigorously with water, and because the concentrations of radioactive materials carried by helium coolant can be maintained at a much lower level than in lithium coolant, intermediate coolant systems are not considered necessary to reduce releases of radioactive materials for designs employing helium as the primary coolant.

Systems using molten sodium or NaK as intermediate coolant will utilize liquid metal technology developed in the LMFBR program. It is expected that piping, pumps, intermediate heat exchangers and steam generators will be adapted with a minimum of modification. A safety feature of LMFBR design is the use of a positive pressure differential across the IHX to prevent radioactive contamination of the intermediate coolant by the primary coolant if an IHX leak develops. The pressure of the intermediate side in the IHX is maintained higher than that of the primary coolant, so that leakage is always from the intermediate to the primary coolant.

In the UWMAK-I design provision is made for the storage of a fraction of the sodium flow during periods of operation, for circulation to the steam generators during the hourly shutdowns. Use of this "thermal flywheel" is expected to allow continuous electrical generation despite the fact that Tokamaks cannot operate continuously. In the PPPL Tokamak the flibe in the blanket acts as a similar heat reservoir. In the theta pinch and laser-pellet designs the pulse period is partly determined by the time required to remove heat deposited in the blanket by the thermonuclear pulse; thus operation is continuous.

The potassium liquid/vapor binary system of the ORNL Tokamak design operates by boiling liquid potassium in tube banks located in lithium flow channels within the blanket, piping the vapor from the blanket to a topping turbine used to drive an electrical generator, and then using the turbine exhaust to raise steam for additional electrical generation. Liquid potassium is pumped back into the blanket by a turbine driven feed pump. This is an advanced concept for which technology may be available when fusion power plants are built.

Potassium vapor is not subject to MHD losses as it crosses magnetic field lines in leaving the blanket. Although MHD losses do occur when liquid potassium is pumped into the blanket, the heat removal capacity of boiling potassium exceeds that of liquid lithium. It is expected that use of this system will reduce pumping losses by more than a factor of ten below those expected when lithium is pumped from the blanket.

Helium is the intermediate coolant in the mirror design. The system in which this coolant circulates has not been designed, except for the Li-He intermediate heat exchanger described above. Steam Generators - In all of the designs steam is generated to drive turbine generators. Steam generator designs for systems using liquid metal intermediate coolants are adapted from 
designs developed in the LMFBR program. These steam generators are designed not only to transfer heat efficiently, but also to minimize the consequences of liquid metal/water reactions by providing a sturdy containment shell and by allowing the relief of excessive pressure by rupture disks.

To inhibit the diffusion of tritium, the fusion power plant adaptation of LMFBR steam generators may also incorporate additional design features. Double-walled construction has been proposed, with a 25-millimeter helium-filled gap separating the walls. Trace amounts of oxygen are to be maintained in the helium to react with the tritium diffusing into it. Metal composite construction has also been proposed with tritium diffusion inhibited by a layer of copper sandwiched within steam generator tube walls.

Steam generator designs for systems using helium primary coolant are adapted from designs used in HTGRs. Helical-coil counter flow designs are used to achieve efficient heat transfer surfaces and to minimize capital costs. Steam generators are located outside the blanket, in contrast to HTGRs where they are located in cavities within the prestressed concrete reactor vesse1. External location and the use of metal rather than concrete pressure containment al low greater design flexibility for fusion power plant steam generators.

Prevention of tritium contamination of the steam system due to steam generator leaks is achieved in the PPPL design by maintaining steam pressure higher than helium pressure. The tritium content of the helium is controlled by continuous removal of tritium which diffuses in from the blanket flibe. Consequently, the potential for steam system contamination due to steam generator leaks is reduced, and the diffusion of tritium across steam generator walls during normal operation is reduced as well. The possibility of further reducing tritium diffusion into the steam system by using steam generator tubes having an internal copper layer is also considered in the PPPL design.

Heat Dissipation - Steam systems are designed to operate on a closed cycle, requiring steam which has been expanded through the turbines to be condensed for recycle to the feedwater heaters and steam generators. Condensation of exhaust steam takes place via heat exchange to banks of tubes through which cooling water is circulated. Waste heat absorbed by the cooling water is dissipated to the atmosphere by cooling towers in all designs which are sufficiently complete to discuss heat dissipation. A variety of types of cooling towers (wet or dry, natural or forced draft) are used.

\subsection{Electricity Generation Systems}

All power plant designs utilize steam turbine-generator systems for the generation of electricity. The ORNL Tokamak design incorporates a topping cycle in which potassium vapor is used to drive a turbine-generator; then steam is generated in the potassium condenser. Direct conversion, a process whereby the kinetic energy of charged particles is converted directly to electrical energy, is utilized in the mirror and theta pinch designs. 
Steam Turbine-Generator Systems - Due to the extensive use of these systems in fossil and fissile fueled power plants, a commercial industry exists for their design and production. Two of the designs encompassed by the reference envelope were actually prepared by commercial vendors for specific steam conditions. Other designs use modifications of systems, either existing or proposed, which were designed by vendors. Power plant designs which are incomplete most often lack the turbine-generator design. In general this is because the designers chose to devote more time to the development of systems less well understood.

The turbine-generator systems use separate high, intermediate and low pressure turbines grouped in various combinations on generator drive shafts. Steam is routed sequentially through the turbines, passing through moisture separators in between. In most designs steam reheat between turbines is also employed, to improve steam cycle efficiency.

The generators are either single or double-wound units, operating at 3600 or 1800 revolutions per minute. They generate 60 cycle, three-phase electrical power at about 20 kilovolts. The generators are filled and cooled with hydrogen gas, which is contained by a seal oil system. Generator outputs are around $1000 \mathrm{MWe}$, and generator designs are generally within the current state-of-the-art.

The ORNL potassium topping cycle turbine-generator system will produce an electrical output which is two-thirds as large as that of the following steam turbine-generator system. The potassium system fuel pump will be driven by a turbine turned by potassium vapor extracted from the topping turbine. Exhaust vapor from the topping turbine will be condensed in a steam generator which supplies superheated steam to the steam turbines, plus two stages of steam reheat. Tritium permeation into the turbine hall from the potassium vapor turbine will be minimized by encasing the turbine in an evacuated stainless steel housing.

Direct Convertor Systems - Direct conversion of plasma energy to electrical power takes place during the theta pinch burn. Fusion energy deposited in the plasma by alpha particles heats the plasma, causing it to expand. This expansion does work against the confining magnetic field, which is converted directly into electrical energy in the circuits which power the theta pinch compression coil.

In the mirror design, plasma particles escape selectively through one of the mirror regions of the magnetic confinement field. These particles are channeled by magnetic fields into a direct convertor structure located outside of the reactor. This structure is larger than the reactor, as may be seen in Figure 26.

The direct convertor has two regions. Escaping plasma ions first enter a magnetic expander region, where the magnetic field lines (and the ions spiraling along them) are fanned out. Then they enter a collector region, where ion kinetic energy is converted to electrical energy. The magnetic expander uses the same basic principle that makes mirror confinement possible. Specifically, an ion spiraling along a magnetic field line in a region where the field strength is changing experiences a force directed away from the high field region. The magnetic field weakens as it is fanned out in the expander, and consequently the ions are accelerated toward the collector region. Thus, the expander maximizes the recoverable kinetic energy of the ions. 

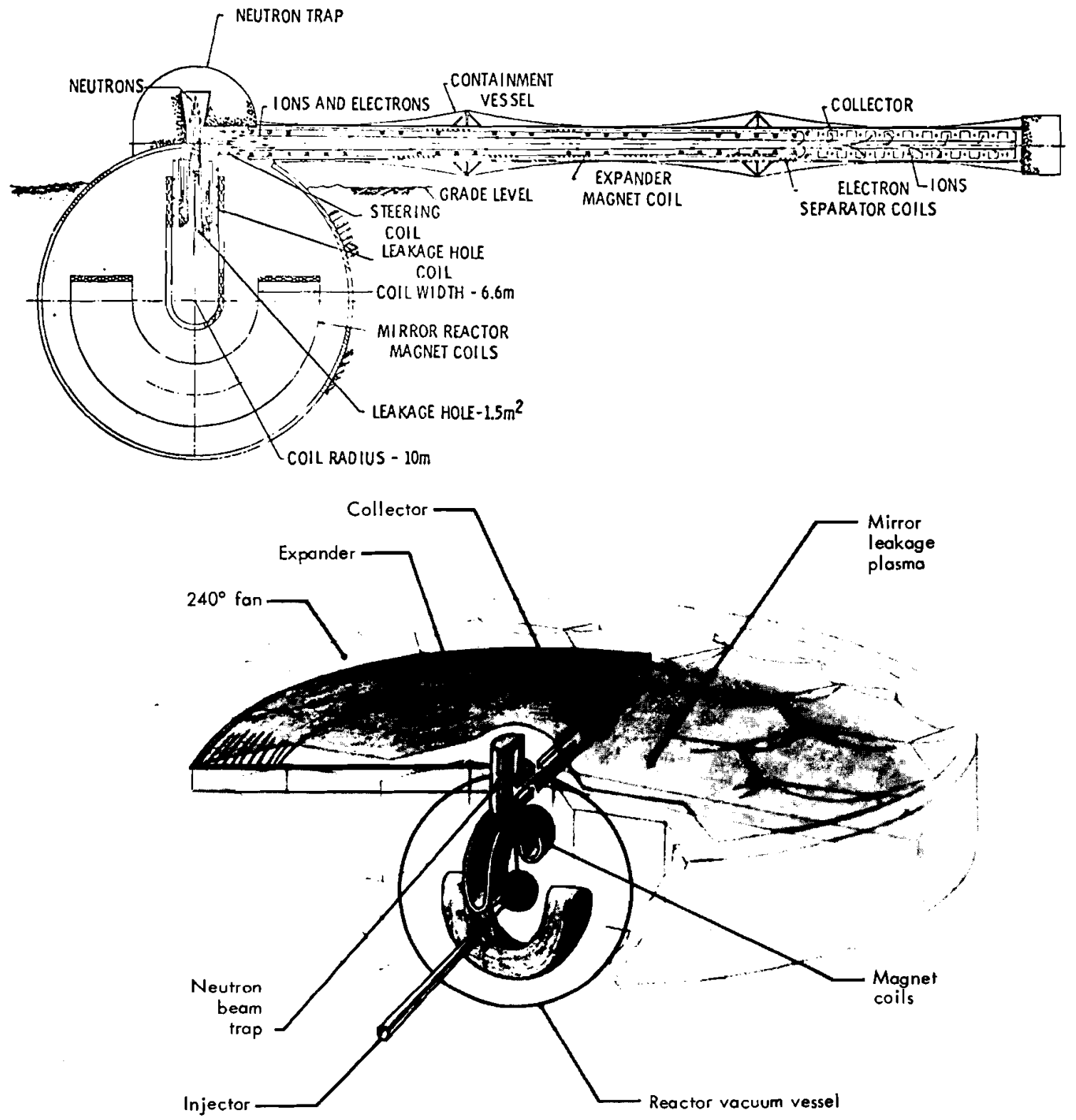

FIGURE 26 Mirror Reactor and Direct Convertor Structure in Section and Perspective Views

In the collector region of the direct convertor the ions encounter strong electric fields established by high voltage electrode structures. The ions are slowed by the electric fields, and their kinetic energy is converted to electrostatic potential energy. When the slowed ions are captured and neutralized by collector electrodes, their electrostatic potential energy becomes directly available to power electric circuits connected to the electrodes. Thus, the kinetic energy of ion motion is converted directly to electrical power without the use of an intermediate thermal cycle and its attendant limitations on conversion efficiency. 


\subsection{Spent Plasma Collection Systems}

Two basic methods for collecting spent plasma are employed - vacuum pumping, and entrainment in liquid lithium. These methods are employed singly and in combination. They are supplemented in some cases by the use of magnetic fields to channel ions escaping from the plasma into collection chambers.

Vacuum Pumping - The most straightforward method of collecting spent plasma is to wait until the thermonuclear burn is quenched and the plasma is neutralized, and then to pump it as a gas from the plasma chamber with vacuum pumps. This method is employed in the ORNL Tokamak and in the theta pinch designs. In the latter, vacuum pumping is supplemented by purging the plasma chamber with fresh fuel gas. Vacuum pumping during the Tokamak burn is also employed to remove plasma which has escaped confinement and has been neutralized at the chamber wall.

In the mirror design, ions which escape the plasma during the thermonuclear burn are channeled by magnetic field lines into a direct convertor. These ions are collected on electrodes, where they are neutralized and reemitted as neutral gas atoms. Vacuum pumps then collect this gas.

Magnetic divertors are included in the PPPL and UWMAK Tokamak designs, to protect plasma chamber walls from bombardment by energetic ions escaping the plasma. The toroidal magnetic confinement field is modified to divert ions which leave inner plasma regions into collection chambers. In the PPPL design the plasma cools and deionizes as it enters the collection chamber, and the neutral gas is exhausted by vacuum pumps.

Mercury diffusion pumps and cryopumps are used for designs requiring plasma chamber pressures lower than $10^{-3}$ torr. Mercury is the diffusion pump working fluid instead of oil, to avoid hydrogenation of pump oil by tritium. Cryopumps are used in addition to diffusion pumps in designs requiring pressures lower than $10^{-4}$ torr. Cryopumps use liquid helium-cooled charcoal to absorb gas atoms which contact the charcoal. They are operated in pairs, with one pump absorbing and the other being regenerated by warming the charcoal.

Entrainment in Liquid Lithium - The magnetic divertors of the UWMAK-I and UWMAK-II designs cause the ions which they collect to impact a flowing stream of liquid lithium. Most of these ions are trapped in the lithium because of the great affinity between liquid lithium and hydrogen isotopes. Recovery of unburned fuel for recycle to the plasma is accomplished by systems similar to those which recover bred tritium from blanket lithium. In these designs the lithium also functions to prevent erosion of collection chamber surfaces by ion impacts, and to carry off the energy of the collected ions.

In all three of the inertial confinement designs, particles and radiation emanating from the fusion microexplosions are deposited in a first surface of liquid lithium. In the Wetted Wall design this causes considerable ablation of the lithium surface, while in the Blascon and Suppressed Ablation designs blowoff of the lithium surface is minimized to reduce shock waves induced in the blanket. However, in all three designs the unburned fuel becomes entrained in the lithium and travels with it for subsequent recovery along with tritium bred in the lithium. In some of these designs vacuum pumping between fusion pulses removes lithium vapor and noncondensable reaction products. 


\subsection{Bred Tritium Recovery Systems}

A variety of methods are proposed to recover bred tritium. Discussions of these methods are grouped according to whether tritium is extracted from the fertile material by cold trapping, gettering, permeation or diffusion.

Cold Trapping - Tritium bred in liquid lithium forms LiT, which dissolves in the liquid lithium. Because the solubility of LiT in lithium is temperature dependent, LiT can be separated from the liquid lithium by crystallizing it in cold traps. This method is employed to remove bred tritium from the lithium primary coolant of the Wetted Wall laser-pellet design. A side stream is drawn from the cold leg of the primary coolant loop and passed through a cold trap, where the LiT crystallizes and is deposited. The LiT is subsequently decomposed by heating $i t$, and the tritium is recovered as a gas.

Gettering - In the UWMAK-I design, tritium is recovered from a side stream of liquid lithium taken from the cold leg of the primary coolant system. This lithium is circulated through a bed of yttrium foil, which forms hydrides more stable than LiH. Consequently, tritium is preferentially absorbed by the yttrium, and the lithium is returned to the coolant system depleted in tritium. When sufficient tritium has accumulated in the yttrium foil, the lithium flow is diverted to an alternate bed. The saturated bed is regenerated by heating it after draining the lithium from it. Tritium is evolved from the heated yttrium as a gas, and is pumped to storage.

In the theta pinch design, tritium is also recovered from a side stream of liquid lithium taken from the cold leg of the primary coolant system. In this design, however, a liquid gettering agent is employed. The liquid lithium is intimately mixed with an equal amount of the molten salt, LiCl, which has a greater affinity for dissolved LiT than does the lithium. The mixture is then separated by centrifugation, with about six times as much LiT going with the salt as with the lithium. Separation of the tritium from the salt may be accomplished either electrochemically or by sparging with $D C 1$, using the reaction $D C 1+L i T \rightarrow L i C 1+D T$.

Permeation - Tritium is separated from liquid lithium primary coolant by permeation through the metal coolant containment in both the ORNL Tokamak and the mirror designs. In the mirror design the blanket is composed of lithium-carrying stainless steel tubes, and the tritium simply permeates through the tubes into the evacuated reactor she11, for collection by the vacuum system. Coating the stainless steel tubes with a refractory oxide such as alumina suppresses tritium permeation in the heat exchanger region.

In the ORNL Tokamak design tritium permeates primarily through the niobium tubes which exchange heat to the potassium intermediate coolant. Tritium is recovered from the potassium by liquid gettering (1iquid lithium is dissolved in the potassium condensate tank and binds the tritium as $L i T)$. A side stream from the condensate tank is passed through a cold trap where the LiT crystallizes and is filtered from the potassium flow. The LiT is subsequently decomposed by heating it, and the tritium is recovered as a gas. In addition, some tritium is expected to permeate directly through the niobium walls of the blanket to the vacuum system. 
Diffusion - The PPPL and UWMAK-II Tokamak designs and the BNL Tokamak blanket design recover tritium by diffusion from surfaces of the material in which it is bred.

In the PPPL Tokamak design the fertile material in the blanket is flibe, a eutectic mixture of the molten salts $\mathrm{LiF}$ and $\mathrm{BeF}_{2}$. Neutron interactions with the lithium produce tritium and simultaneously liberate fluorine, which then combines with the tritium to form tritium fluoride (TF). Tritium recovery is accomplished by spraying the flibe into an evacuated tank, forming tiny droplets from which the TF diffuses before the spray is collected in the bottom of the tank for return to the blanket. The offgas from the spray is cooled to trap out entrained salt mist, then further cooled to liquid nitrogen temperatures to condense the TF and separate it from helium which is also produced in the flibe by neutron reactions on fluorine. The tritium is separated from the TF by electrolyzing a solution of TF dissolved in $\mathrm{KCl}$. Tritium is liberated as a gas for recycle to the fuel system.

In the UWMAK-II design, tritium is produced in microspheres of $\mathrm{LiAlO}_{2}$, which are contained in stainiess steel tubes. The tubes are connected to plenna for the direct collection of tritium gas which diffuses from the microspheres.

In the BNL blanket design, tritium is produced in microspheres of $\mathrm{LIAl}$ and $\mathrm{LiAlO}_{2}$ which are constituents of larger porous balls. The helium blanket coolant flows directly over the balls, and the tritium diffuses into the coolant stream. A sidestream is diverted from the cold leg of the coolant system and is directed through beds containing fine particles of titanium metal getter and inert ballast. The tritium is absorbed by the titanium, and then released by outgassing at the same temperature into a high vacuum maintained by cryopumps which collect the tritium gas for transmission to the fuel system. 


\section{CHARACTERIZATION OF THE REFERENCE POWER PLANTS}

The characteristics attributed to the reference power plants are those expected to cause significant environmental interactions in addition to those interactions common to all thermal power plants. They are determined by analyzing the envelope of D-T fusion power plant characteristics to identify which could cause significant environmental interactions. The sixteen primary power plant environmental interactions are considered: use of land, labor and materials; release of heat, chemicals, sanitary wastes and liquids; production of noises, vibrations, odors and magnetic fields, disposal of solid, gaseous and radioactive wastes, aesthetics, and material transportation.

No design concept has yet been published for an advanced fusion power plant. Consequently, possible characteristics of such a plant are determined by reexamining the envelope of current D-T fusion power plant characteristics, using the assumption that the fusion reaction employed neither creates nor consumes radionuclides. Characteristics expected to cause significant environmental interactions were then determined for the Reference Advanced Commercial Fusion Power Plant.

\subsection{Power Plant Environmental Interactions}

The primary types of power plant environmental interactions are identified in Table 3, along with environmental changes that they cause. Figure 27 provides a pictorial example of these interactions. For each of these interactions the envelope of D-T fusion power plant characteristics was examined to determine those which could cause significant interactions.

Some environmental interactions are common to all power plants, and are easily controlled by use of current best technology. An example is release of sanitary wastes. Such sources of interactions are identified as being common to all thermal power plant environmental analyses and are not included in descriptions of the reference power plants, because these interactions will automatically be considered in any power plant environmental analysis. A tabulation of these common characteristics is presented at the end of the next subsection.

Land Use - The land required for a power system generally is determined by four factors: 1) the area occupied by structures and facilities, 2) the exclusion areas needed to prevent significant impact on the general public as a result of aesthetic effects or releases of pollutants and noises, 3) the plant isolation necessary to prevent damage to the plant as a result of actions by the general public, and 4) the land used by separate fuel cycle supporting facilities such as mines and fuel fabrication plants.

The amount of land required for providing construction materials is not considered here. It is determined through an evaluation of material requirements and is considered under material use. (13)

The envelope of fusion power plant characteristics includes four primary determinants of the amount of land that would be required by a fusion power plant: 1) the land occupied by the plant structures and facilities, 2) the waste heat disposal system, 3) the exclusion area needed because of the presence and potential for release of radionuclides and chemicals and 4) the exclusion area needed to prevent contact of the general public with strong magnetic fields. 
TABLE 3 Typical Power Plant Environmental Interactions

\begin{tabular}{|c|c|}
\hline Environmental Interaction & Resultant Significant Environmental Changes \\
\hline Land Use & $\begin{array}{l}\text { Human Displacement } \\
\text { Animal Displacement } \\
\text { Change in Vegetation } \\
\text { Foreclosed Uses }\end{array}$ \\
\hline Labor Usage & Societal Changes \\
\hline Material Usage & $\begin{array}{l}\text { Environmental Effects During Material Procurement } \\
\text { Foreclosed Uses }\end{array}$ \\
\hline Heat Releases & $\begin{array}{l}\text { Atmospheric Effects } \\
\text { Changes in Life Forms }\end{array}$ \\
\hline Chemical Releases & Changes in Life Forms \\
\hline Sanitary Waste Release & Changes in Life Forms \\
\hline Noise Release & Degradation of Living Conditions \\
\hline Vibrations & $\begin{array}{l}\text { Structural Damage } \\
\text { Degradation of Living Conditions }\end{array}$ \\
\hline Odors & Degradation of Living Conditions \\
\hline Magnetic Fields & Biological Changes and Electrical Effects \\
\hline Liquid Releases & $\begin{array}{l}\text { Land Structure Changes } \\
\text { Mechanical Damage to Life Forms } \\
\text { Chemical Damage to Life Forms }\end{array}$ \\
\hline Solid Waste Disposal & Land Use Impacts \\
\hline Gaseous Waste Disposal & $\begin{array}{l}\text { Change in Life Forms } \\
\text { Degradation of Living Conditions }\end{array}$ \\
\hline Radionuclide Disposal & $\begin{array}{l}\text { Land Use Impacts } \\
\text { Biological Damage }\end{array}$ \\
\hline Aesthetic Changes & Degradation of Social Conditions \\
\hline Material Transportation & $\begin{array}{l}\text { Noise Impacts } \\
\text { Chemical Release Impacts } \\
\text { Radiation Dose Impacts }\end{array}$ \\
\hline
\end{tabular}

Land requirements for plant structures, facilities, and waste heat disposal systems are similar for all fusion power plant design concepts and for other types of power plants.

The exclusion area needed because of the presence of radionuclides and chemicals is determined by the following characteristics:

1. The $D-T$ fusion reaction,

2. The presence of large quantities of activation products,

3. The presence of kilogram quantities of tritium,

4. The presence of large quantities of potentially hazardous chemicals such as lithium and sodium. 


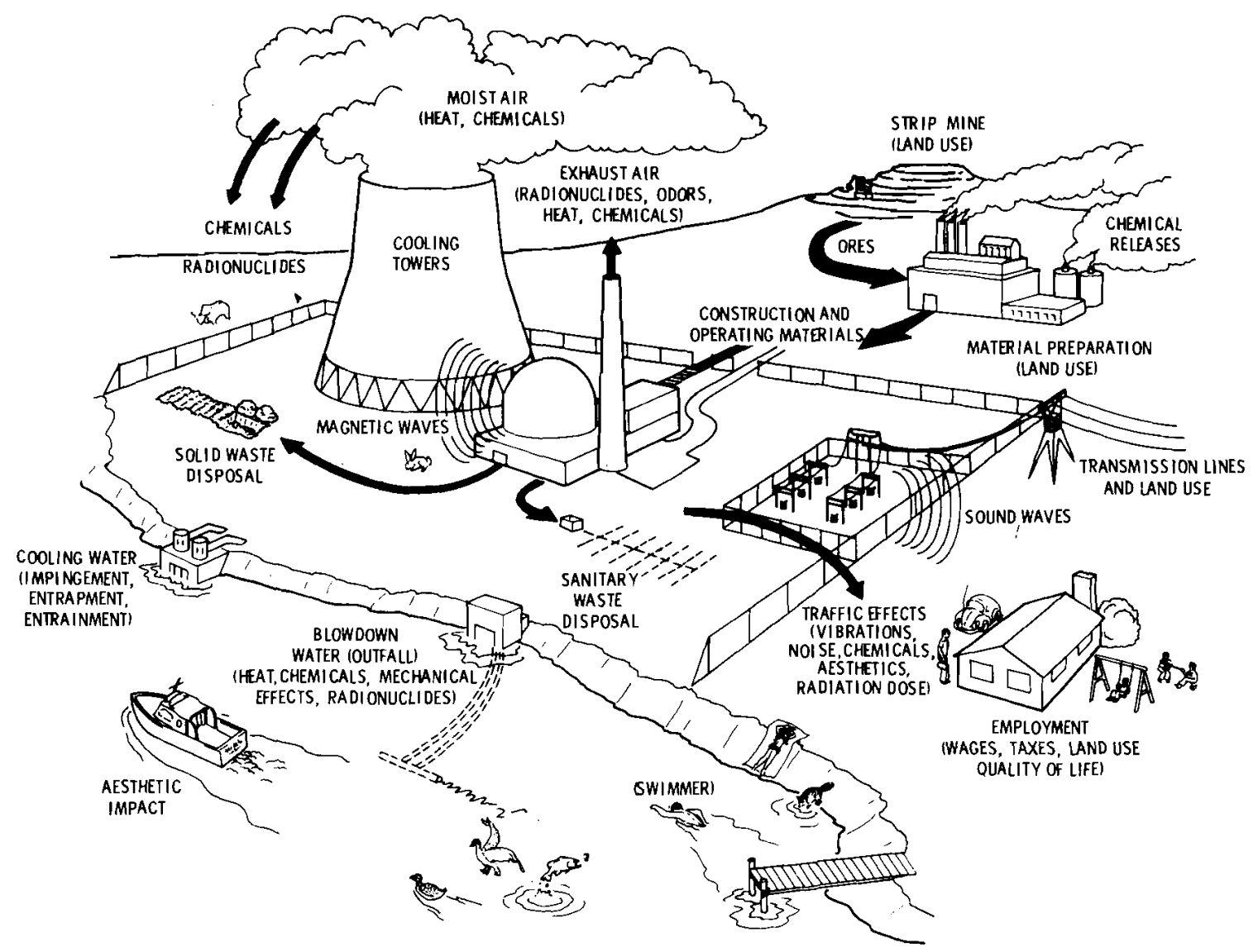

FIGURE 27 Typical Power Plant Environmental Interactions

An exclusion area is necessary to prevent contact of the general public with strong magnetic fields present in some design concepts. Such fields probably would interfere with communications and other electrical systems. Although such fields will not occur in all fusion power plant design concepts, the magnetically confined reactors currently are the primary candidates for the first commercial power plants. Consequently, the presence of large magnetic fields is included as a characteristic of the reference first commercial fusion power plant to assure that all potentially significant environmental effects are considered.

Fusion power plants have no significant requirements for offsite facilities for the fuel cycle. The fuel cycle facilities are contained in the power plant (in contrast to coal-fired power plants that have extensive land use for providing coal and to fission plants that require uranium mining, enrichment, fuel fabrication, reprocessing, and radioactive waste storage). A11 fuel materials (deuterium and $7 i$ thium) used during normal operation are expected to be purchased as raw materials from the open market and in relatively small quantities that will not have a significant effect on the supply industries. Thus, the use of a self-contained fuel cycle limits land use primarily to that at the power plant site.

Labor Use - All fusion power plants will require labor forces for construction, operation, maintenance, and decommissioning. This is a common requirement for all power plants and will not be included in the reference design. 
Material Use - All fusion power plant design concepts appear to be much more complex and larger than current fossil and fissile power plants of the same capacity. This complexity results from the need for massive blankets and shields to contain energetic neutrons and generate tritium, the use of large and numerous magnets to provide magnetic fields, the low average power density in the reactor blanket, and the need for intermediate heat exchange systems to isolate primary coolants from the steam system. In addition, the generation of tritium for fuel requires large inventories of 1 ithium in all concepts and of neutron multipliers in some concepts. A supply of deuterium fuel is needed for all concepts.

A11 fusion power plant concepts have standard steam turbine generator systems to produce electricity from the heat energy released when neutrons are moderated and captured. These require the normal massive waste heat disposal systems. The standard electricity generation systems are common to all thermal plants.

Analys is shows that the primary plant characteristics that determine the quantities of materials needed for construction and operation of the first fusion power plants are:

1. The D-T fusion reaction,

2. The massive reactor structures,

3. The large lithium inventories,

4. The large magnetic fields, and

5. The use of standard electricity generation systems.

Heat Releases - A11 D-T fueled fusion power plant design concepts generate heat by the moderation and capture of energetic neutrons and use standard steam-powered turbine generator systems. The resultant release of waste heat to the environment is common to all thermal power plants and is considered in environmental analyses.

Chemical Releases - The primary chemical releases from D-T fueled fusion power plants are expected to be 1) routine releases of biocides and corrosion inhibitors in the waste heat disposal waste water streams and 2) possibly the release of liquid metal coolants such as lithium or sodium during accidents. The technology of waste treatment of liquid and gaseous waste streams currently is good enough that releases of significant quantities of chemicals other than those in the waste heat disposal waste water streams can be prevented during routine operations. Accident situations may result in significant releases of chemicals present in large quantities in spite of application of best current technology. Accidental releases of chemicals present in small quantities generally can be prevented or generally result in insignificant environmental effects.

The release of chemicals from the waste heat disposal system is common to all thermal power plants and will be characterized in the reference plants by noting that they have a standard electricity generation system.

Any accidental release of significant quantities of chemicals is expected to be due solely to the presence of liquid metals or salts in the primary or secondary cooling systems. 
Sanitary Waste Releases - All power plants have sanitary waste releases, and ample technology is available to prevent significant adverse environmental effects. They will not be considered in characterizing the reference plants.

Noise Releases - The primary causes of significant noises at an operating power plant are exterior mechanical equipment for material handling, electric motor hum, and transformer hum. All major construction projects cause many noises due to operation of heavy equipment, movement of materials, and blasting. The causes of noises during construction will not be considered in characterizing the reference plants because they are common to all designs.

Noises due to material handling are not expected to be significant at an operating fusion power plant because only incidental amounts of material must be handled. Motor and transformer noises are common to all power plants and can be reduced to an insignificant level by use of current control technology. Therefore, they will not be considered in characterizing the reference plants.

Vibrations - No significant source of vibrations has been identified for an operating fusion reactor. Vibrations may occur during construction if blasting is necessary but this is common to all construction jobs.

Significant vibrations during fusion reactor operation probably cannot be tolerated because of the large amounts of equipment requiring precise operation (e.g., lasers and neutral beam injectors). As a result, no plant characteristics that result in vibrations have been identified.

Odors - No significant source of odors has been identified for operating fusion power plants. odors may occur during construction (installation of tar roofs, etc.) but this is common to all construction jobs.

Significant quanticies of odoriferous materials are not included in the current D-T fueled fusion reactor design concepts. In addition, ample technology currently is available for preventing routine releases of such materials. As a result no plant characteristics that would result in significant releases of odors have been identified.

Magnetic Fields - Large magnetic fields are needed for many fusion reactor design concepts and will be included in the reference first power plant description to assure that all significant interactions with the environment will be considered.

Liquid Releases - The primary liquid releases from a nuclear power plant are sanitary wastes, service water, and cooling system blowdown or once-through cooling streams. The sanitary waste stream is discussed above. The service water stream usually contains only waste heat, is common to all power plants, and as a result will not be considered in characterizing the reference plants. The blowdown or once-through stream from the cooling system is common to all thermal power plants and does not have to be considered as a separate unique item.

Solid Waste Disposal - All power plants create miscellaneous nonradioactive solid wastes that are disposed of by incineration or shipment to landfill sites. Since no unique solid wastes are apparent for a fusion power plant, solid wastes are considered to be a common characteristic. 
Gaseous Waste Disposal - The only apparent gaseous wastes for fusion power plants are the exhaust gases from the heating boiler and the emergency diesel generator. These are common to all nuclear power plants. It is possible that various organic solvents may be used in fuel preparation activities, but ample technology is available for preventing significant releases to the environment. No such materials have been identified.

Radionuclide Disposal - A11 current design concepts use the D-T fusion reaction. This creates numerous neutrons and activation products. In addition, tritium will be present in many parts of the power plant. As a result, solid, liquid, and gaseous radwaste systems will be necessary characteristics of the reference first commercial fusion power plant.

Aesthetic Effects - The fusion power plant is visualized as being a large reactor building with the associated supporting structures such as a turbine-generator building and cooling towers. The external appearance of these structures is expected to be the same as for current nuclear power plants although the dimensions may be larger. The aesthetic impact should be similar and will be treated as a common characteristic of nuclear power plants.

Material Transportation - The primary materials transported for a fusion power plant appear to be 1) the normal construction materials, 2) small amounts of fuel materials (deuterium, bery1lium, lithium compounds), 3) a tritium inventory for plant startup, and 4) solid radioactive wastes. This transportation appears to be similar to that of current nuclear power plants except that there does not appear to be any transportation of 1) massive reactor pressure vessels (up to 1200 tons for LWRs) or 2) biologically significant radionuclides such as plutonium and fission products. Transportation of highly radioactive spent inner wall materials to burial grounds may be equivalent to the transportation of irradiated fuel elements from fission power plants.

The D-T fusion power plant design characteristics that define the need for transportation of radioactive wastes are:

1. The D-T fusion reaction,

2. The presence of large quantities of activation products,

3. The presence of kilogram quantities of tritium, and

4. The use of standard radwaste systems to accumulate radioactive wastes for shipment offsite for disposal.

Analysis Summary - The results of this analysis of the envelope of D-T fusion power plant characteristics to identify which would cause significant environmental interactions is summarized in Table 4. Each of the sixteen normal power plant primary environmental interactions is listed; and the fusion reactor characteristics resulting in these types of interactions are described.

\subsection{The Reference First Commercial Fusion Power Plant}

As a result of the analysis of the envelope of current D-T fueled fusion power plant characteristics, unique environmentai interactions are expected to be caused by these characteristics attributed to the reference first commercial fusion power plant: 
TABLE 4 Causes of Fusion Power Plant Environmental Interactions

\begin{tabular}{|c|c|}
\hline $\begin{array}{l}\text { Environmental } \\
\text { Interaction } \\
\end{array}$ & $\begin{array}{l}\text { Causes Common To All } \\
\text { Thermal Power Plants }\end{array}$ \\
\hline Land Use & $\begin{array}{l}\text { Land occupancy by structures and cooling } \\
\text { system } \\
\text { Land disturbed during material } \\
\text { procurement }\end{array}$ \\
\hline Labor Use & $\begin{array}{l}\text { Construction force } \\
\text { Operations force }\end{array}$ \\
\hline Material Use & Standard electricity generation systems \\
\hline Heat Releases & Standard electricity generation systems \\
\hline Chemical Releases & Standard electricity generation systems \\
\hline Sanitary Wastes & Sanitary waste releases \\
\hline Noises & $\begin{array}{l}\text { Construction activity } \\
\text { Motor hums } \\
\text { Transformer hums }\end{array}$ \\
\hline Vibrations & Construction activity \\
\hline Odors & Construction activity \\
\hline Magnetic Fields & Normal electrical systems \\
\hline Liquid Releases & $\begin{array}{l}\text { Sanitary waste } \\
\text { Service water } \\
\text { Cooling system waste streams }\end{array}$ \\
\hline $\begin{array}{l}\text { Solid Waste } \\
\text { Disposal }\end{array}$ & $\begin{array}{l}\text { Construction waste } \\
\text { Normal operating wastes }\end{array}$ \\
\hline $\begin{array}{l}\text { Gaseous Waste } \\
\text { Disposal }\end{array}$ & $\begin{array}{l}\text { Heating boilers } \\
\text { Emergency diesel generators }\end{array}$ \\
\hline $\begin{array}{l}\text { Radionuclide } \\
\text { Disposal }\end{array}$ & None \\
\hline Aesthetic Effects & $\begin{array}{l}\text { Construction activities } \\
\text { Industrial structures }\end{array}$ \\
\hline $\begin{array}{l}\text { Material } \\
\text { Transportation }\end{array}$ & $\begin{array}{l}\text { Construction materials } \\
\text { Solid wastes } \\
\text { Decommissioning }\end{array}$ \\
\hline
\end{tabular}

D-T Fusion Power Plant Characteristics Causing Unique Environmental Interactions

The D-T fusion reaction.

Large quantities of activation products.

Kilogram quantities of tritium.

Large quantities of potentially

hazardous liquid metals.

Self-contained fuel cycle.

Rural siting.

None apparent.

The $D-T$ fusion reaction.

Massive reactor shield and blanket.

Large lithium inventories.

Large magnetic fields.

None apparent.

Liquid metal coolants.

None apparent.

None apparent.

None apparent.

None apparent.

Large magnetic fields.

None apparent.

None apparent.

None apparent.

The D-T fusion reaction.

Large quantities of activation products. Kilogram quantities of tritium.

None apparent.

The D-T fusion reaction.

Large quantities of activation products. Kilogram quantities of tritium.

Standard radwaste systems. 
- The D-T fusion reaction

- Large quantities of activation products in plant

- Kilogram quantities of tritium in plant systems

- Massive reactor blanket and shield structures

- Large lithium inventories

- Large inventories of liquid metals or salts

- Standard electricity generation systems

- Standard radwaste systems

- Large magnetic fields

- Self-contained fuel cycle

- Rural siting

Standard electricity generation systems are included in the above list despite the fact that they are common to all thermal generation plants. This system defines many of the environmental interactions (land use, heat releases, chemical releases, noises, liquid releases, and aes thetics).

Characteristics of the reference first commercial fusion power plant which are common to all thermal power plants are:

- Land occupancy by structures

- Land occupancy by cooling systems

- Land disturbance during material procurement

- Construction force and activities

- Operating force

- Standard electricity generation system

- Sanitary waste releases

- Motor hums

- Transformer hums
- Normal electrical systems

- Service water usage

- Construction wastes

- Operation solid wastes

- Heating boilers

- Emergency diesel generators

- Industrial structure aesthetic effects

- Construction material transportation

- Solid waste transportation

- Decommissioning waste transportation

\subsection{The Reference Advanced Commercial Fusion Power Plant}

The advanced fusion power plants are visualized as operating with a fusion reaction that neither uses radionuclides as fuel nor creates radionuclides as reaction products or through neutron activation of structure or coolant materials. That fusion reaction is expected to have much more severe operating condition than will the reactions expected to be used in the first commercial power plants. The method of confinement for such an advanced reaction is not known.

Although the fusion reactions used in the advanced reactors are not known they could be any one of many reactions. Typical examples are the proton-lithium reaction,

$$
{ }^{1} \mathrm{H}+{ }^{6} \mathrm{Li} \rightarrow{ }^{3} \mathrm{He}+{ }^{4} \mathrm{He}+4.0 \mathrm{MEV}
$$

or the proton-boron reaction

$$
1_{H}+1_{B} \longrightarrow 3^{4} \mathrm{He}+8.7 \mathrm{MEV}
$$

The fusion reactions used in the advanced reactors probably will produce many types of radiation such as alpha, beta, and gamma rays, but it is assumed that an insignificant number of neutrons 
would be generated. Shielding would be necessary for stopping the gamma rays, but this need only be massive materials such as concrete with embedded cooling tubes.

Technology will be much more advanced when these power plants are operating, and it is assumed that direct conversion generation of electricity will be used to increase generating efficiency. However, it is not possible to visualize how all energy produced in the reactor can be converted directly to electricity. Consequently, it is assumed that there still will be inefficiencies resulting in production of large quantities of heat, the use of standard turbine-generators to produce electricity and the release of waste heat. It may be possible to use the waste heat for building heating and cooling but even those processes reject heat - particularly in the summer.

On the basis of this description and the information developed in the analysis of the envelope of D-T fusion power plant characteristics, the reference advanced fusion power plant probably would have the following general characteristics:

- A fusion reaction that neither consumes nor creates radionuclides,

- Minimal radionuclide production in the power plant,

- A reactor of moderate size,

- Standard and advanced electricity generating systems,

- A self-contained fuel cycle, and

- Possible city siting.

Possible city siting would depend on 1) whether large magnetic fields which would have electrical effects on nearby communications systems surround the reactor and 2) whether there would be a waste heat disposal problem. Inertial confinement would eliminate any magnetic field problem. A satisfactory solution to the waste heat problem would depend on ability to use the waste heat for district heating and cooling, and on the availability of other methods for waste heat disposal. 


\section{FUTURE RESEARCH REQUIREMENTS}

The quality of the reference design selected for the commercial fusion power plants is dependent primarily on the completeness and representativeness of the design concepts on which the reference design is based. No specific additional research is required for making the current design concepts more representative of the first commercial fusion power plants other than to continue development until a feasible design is available.

Development of the reference designs of this study, however, did reveal that the current design concepts were not complete enough to permit development of a detailed reference design. Although the descriptions of the fusion reactors generally were adequate, detailed designs of the supporting subsystems generally were very conceptual or did not exist at all.

Since the supporting subsystems determine the interactions with the environment, detailed descriptions are necessary before the environmental effects can be determined. Two methods are available for obtaining those subsystem designs:

1. Develop the subsystem designs for each design concept, or

2. Develop a detailed reference design for the first commercial fusion power plants.

Because all current design concepts indicate similar interactions with the environment (with the notable exception of the size and presence of magnetic fields), the most efficient method for obtaining the subsystem designs appears to be development of a detailed reference design for the first commercial fusion power plant. Development of one detailed design should be less costly than development of ten designs (one for each current design concept) which probably would have quite similar subsystems.

The specific information that should be included in this detailed reference design is as follows:

1. Process flow diagram showing the flow paths and rates of all major materials (fuels, coolants, wastes, etc.),

2. A description of the entire fuel cycle from mine to disposal of the wastes,

3. Sufficient descriptive material so that all radioactive inventories and their flow paths and rates are known,

4. A general description of all major supporting subsystems (waste treatment, ventilation, power generation, etc.),

5. Sufficient descriptions of any equipment that controls the release rates of pollutants so that the release rates can be determined,

6. Any unique siting or construction requirements, and

7. A tabulation of the quantities of each significant construction material showing location and degree of fabrication.

The amount of detail provided when describing the equipment and subsystems depends on the novelty of the design. If commercial systems are available for a function, it is sufficient to 
say that a standard commercial system will be used. On the other hand, if a novel design is proposed, sufficient description must be provided so that the environmental interactions can be estimated.

Any design concepts prepared in the future should contain all of the above information. 


\section{REFERENCES}

1. J. R. Young, An Environmental Analysis of Fusion Power to Determine Related R\&D Needs, BNWL-2010, Battelle, Pacific Northwest Laboratories, Richland, WA, 1976

2. B. F. Gore and E. S. Murphy, Current Fusion Power Plant Design Concepts, BNWL-2013, Battelle, Pacific Northwest Laboratories, Richland, WA, 1976.

3. B. Badger, et a1., (30 authors), UWMAK-I, A Wisconsin Toroidal Fusion Reactor Design, UWFDM-68, University of Wisconsin, Vol. I (1974) and II (1975).

4. B. Bạdger, et al., (30 authors), UWMAK-I I, A Conceptual Tokamak Power Reactor Design, UWFDM-112, University of Wisconsin, Oct 1975.

5. J. R. Powell, Ed., "Preliminary Reference Design of a Fusion Reactor Exhibiting Very Low Residual Radioactivity," BNL-19565, Brookhaven National Lab., Dec 1974.

6. A Fusion Power Plant, R. G. Mills, Ed., MATT-1050, Princeton Plasma Physics Laboratory, 1974.

7. A. P. Fraas, Conceptual Design of the Blanket and Shield Region and Related Systems for a Ful1 Scale Toroidal Fusion Reactor, ORNL-TM-3096, May 1973.

8. An Engineering Design Study of a Reference Theta-Pinch Reactor (RIPR), no author - 26 contributors, LA-5336, ANL-8019, (Jo int report), Vols. I (1974) and II (1975).

9. R. W. Werner, G. A. Carlson, J. Hovingh, J. D. Lee and M. A. Peterson, Progress Report \#2 on the Design Considerations for a Low Power Experimental Mirror Fusion Reactor, UCRL-74054-2, Sep 1973.

10. A. P. Fraas, Conceptual Design of a Series of Laser Fusion Power Plants of 100 to 3000 MW(e), Oak Ridge National Laboratory, Oak Ridge, TN. Paper presented at the 9th Intersociety Energy Conversion Engineering Conference, San Francisco, CA, August 26-30, 1974.

11. J. Williams, T. Merson, F. Finch, F. Schilling, and T. Frank, A Conceptual Laser Controlled Thermonuclear Reactor Power Plant, LA-UR-74-593, Los Alamos Scientific Laboratory, Los Alamos, NM, Apr 1974.

12. J. Hovingh, J. Maniscalco, M. Peterson and R. W. Werner, "The Preliminary Design of a Suppressed Ablation Laser-Induced Fusion Reactor," Proceedings of the First Topical Meeting on the Technology of Controlled Nuclear Fusion, April 16-18, 1974, San Diego, CA, CONF-740402-PT, p. 96.

13. J. N. Hartley, L. E. Erickson, R. L. Engel and T. J. Foley, Materials Availability for Fusion Power Plant Construction, BNWL-2016, Battelle, Pacific Northwest Laboratories, Richland, WA, 1976. 


\section{OFFSITE}

A. A. Churm ERDA Chicago Patent Group

9800 S. Cass Avenue

Argonne, IL 60439

J. W. Beal

ERDA Div, of Magnetic

Fusion Energy

Washington, DC 20545

S. 0. Dean

ERDA Div. of Magnetic

Fusion Energy

Washington, DC 20545

E. E. Kintner

ERDA Div. of Magnetic

Fusion Energy

Washington, DC 20545

J. M. Williams

ERDA Div. of Magnetic

Fusion Energy

Washington, DC 20545

J. N. Grace

ERDA Div. of Magnetic

Fusion Energy

Washington, DC 20545

J. Baublitz

ERDA Div. of Magnetic

Fusion Energy

Washington, DC 20545

3 F. E. Coffman

ERDA Div. of Magnetic Fusion Energy

Washington, DC 20545

J. F. Decker

ERDA Div. of Magnetic

Fusion Energy

Washington, DC 20545

3 K. M. Zwilsky

ERDA Div. of Magnetic

Fusion Energy

Washington, DC 20545

Dr. Philip M. Stone

ERDA Applied Plasma Physics

Program

Washington, DC 20545

G. W. Kuswa

ERDA Div. of Laser Fusion

Washington, DC 20545
OFFSITE

R. Blaunstein

ERDA Div. of Biomedical

and Environmental Research

Washington, DC 20545

H. M. Busey

ERDA Div. of Military

Application

Washington, DC 20545

M. A. Bell

ERDA Div. of Safety

Standards and Compliance

Washington, DC 20545

27 ERDA Technical Information Center

M. S. Kaminsky

Argonne National Laboratory

9700 S. Cass Avenue

Argonne, IL 60439

V. A. Maroni

Argonne National Laboratory

9700 S. Cass Avenue

Argonne, IL 60439

P. M. Persiani

Argonne National Laboratory

9700 S. Cass Avenue

Argonne, IL 60439

M. Petrick

Engineering and Technology

Division

Argonne National Laboratory

9700 S. Cass Avenue

Argonne, IL 60439

W. E. Parkins, Manager

Atomics International

Component Engineering and

Technology Division

North American Rockwell

P. 0. Box 309

Canoga Park, CA 91304

D. Gurinsky

Brookhaven National Laboratory

ERDA Brookhaven Area Office

Upton, NY 11973

H. J. Kouts

Brookhaven National Laboratory

ERDA Brookhaven Area Office

Upton, NY 11973

S. Pearlstein

Brookhaven National Laboratory

ERDA Brookhaven Area Office

Upton, NY 11973

Distr-1
OFFSITE

3. R. Powe 11

Brookhaven National Laboratory ERDA Brookhaven Area Office Upton, NY 11973

A. J. Impink, Jr. Carnegie Melion University Pittsburgh, PA 15213

R. A. Gross

Plasma Research Laboratory

236 SW Mudd Bldg.

Columbia University

New York, NY 10027

W. C. Gough

Electric Power Research Inst.

3412 Hillview Ave.

Palo Alto, CA 94304

G. R. Hopkins

Gulf General Atomic

P.0. Box 1111

San Diego, CA 92112

Zeinab Sabri

Iowa State University

26] Sweeney Hall

Nuclear Engineering Department

Ames, IA 50010

R. Borg

Lawrence Livermore Laboratory

P.0. Box 808

Livermore, CA 94550

T. K. Fowler

Lawrence Livermore Laboratory P.0. Box 808

Livermore, CA 94550

R. Moir

Lawrence Livermore Laboratory

P.0. Box 808

Livermore, CA 94550

A. Carl Haussmann

Lawrence Livermore Laboratory

P.0. Box 808

Livermore, CA 94550

3. Hovingh

Lawrence Livermore Laboratory

P. O. Box 808

Livermore, CA 94550

R. F. Post

Lawrence Livermore Laboratory

P.0. Box 808

Livermore, CA 94550

C. J. Taylor

Lawrence Livermore Laboratory

P.0. Box 808

Livermore, CA 94550 
R. Werner

Lawrence Livermore

Laboratory

P.0. Box 808

Livermore, CA 94550

L. L. Wood

Lawrence Livermore

Laboratory

P.0. Box 808

Livermore, CA 94550

W. Bauer

Division Supervisor

of Physical Research

Sandia Labs Livermore

Livermore, CA 94550

L. Booth

Los Alamos Scientific

Laboratory

CTN Research

P.0. Box 1663

Los Alamos, NM 87544

D. J. Dudziak

Los Alamos Scientific

Laboratory

CTN Research

P.0. Box 1663

Los Alamos, NM 87544

D. B. Henderson

Los Alamos Scientific Laboratory

CTN Research

P.0. Box 1663

Los Alamos, NM 87544

E. L. Kemp

Los Alamos Scientific

Laboratory

CTN Research

P.0. Box 1663

Los Alamos, NM 87544

F. L. Ribe

Los Alamos Scientific

Laboratory

CTN Research

P.0. Box 1663

Los Alamos, NM 87544

L. Stewart

Los Alamos Scientific

Laboratory

CTN Research

P.0. Box 1663

Los Alamos, NM 87544

K. Thomassen

Los Alamos Scientific

Laboratory

CTN Research

P.0. Box 1663

Los Alamos, NM 87544
0 . K. Harling

Massachusetts Institute of

Technology

Cambridge, MA 02139

Bruno Coppi

Department of Physics

Massachusetts Institute of

Technology

Cambridge, MA 02139

L. Lidsky

Dept. of Nuclear Engineering

Massachusetts Institute of

Technology

Cambridge, MA 02139

Norm Rasmussen

Dept. of Nuclear Engineering

Massachusetts Institute of

Technology

Cambridge, MA 02139

David Rose

Massachusetts Institute of

Technology

Cambridge, MA 02139

R. E. Stickney

Mechanical Engineering

Massachusetts Institute of

Technology

Cambridge, MA 02139

J. J. Reinmann

NASA - Lewis Research Center 2100 Bookpark Rd.

Cleveland, $\mathrm{OH} \quad 44135$

Vincent Arp

Nationa 1 Bureau of Standards

Cryogenics Division

Boulder, CO 80302

J. F. Clarke

Oak Ridge Nationa

Laboratory

P.0. Box Y

Oak Ridge, TN 37830

A. P. Fraas

Oak Ridge Nationa 1

Laboratory

P. 0. Box Y

Oak Ridge, TN 37830

J. Rand MCNally, Jr.

Oak Ridge National

Laboratory

P.0. Box Y

Oak Ridge, TN 37830

D. Steiner

Oak Ridge National

Laboratory

P. 0. Box Y

Oak Ridge, TN 37830
J. $S \cot t$

Oak Ridge National Laboratory

P. 0. Box $X$

Oak Ridge, TN 37830

J. Banford

Physics International

2700 Merced St.

San Leandro, CA 94577

R. A. Huse

Public Service Electric

and Gas Co.

80 Park Place

Newark, NJ 07101

M. Gottlieb

Princeton University, PPPL

P.0. Box 451

Princeton, NJ 08540

R. G. Mills

Princeton University

P.0. Box 45i

Princeton, NJ 08540

E. C. Tanner

Princeton University

P.0. Box 451

Princeton, NJ 08540

H. Perkins

Dept. of Chemistry

Princeton University

Princeton, NJ 06540

R. E. Gold

303 Sayre Hall

Forresta 1 Campus

P.0. Box 451

Princeton, NJ 06540

M. Kristiansen

Texas Tech. University

Lubbock, TX 79409

A. F. Haught

United Aircraft Research Lab.

United Aircraft Corporation

East Hartford, CT 06108

L. Levine

U.S. Naval Research Laboratory Washington, DC 20390

C. Z. Serpan, Jr.

U.S. Nava1 Research Laboratory Washington, DC 20390

Francis Chen

University of California

Electronics Research

Laboratory

College of Engineering

Berkeley, CA 94720 
A. J. Lichtenberg University of California Electronics Research Laboratory College of Engineering Berkeley, CA 94720

Dave Okrent

U.C.L.A.

Los Angeles, CA 90024

C. D. Hendricks University of Illinois

Nuclear Engineering

Laboratory

Urbana, IL 61801

G. H. Miley University of Illinois

Nuclear Engineering

Laboratory

Urbana, IL 61801

Terry Kammash University of Michigan Nuclear Engineering Department

Ann Arbor, MI 48105

Dean Abrahamson

University of Minnesota

School of Public

Affairs

Social Science

Building/ 309

Minneapolis, MN 55455

W. G. Davey

University of Texas

Department of Physics

Austin, TX 78712

E. Linn Draper, Jr.

University of Texas

Department of Physics

Austin, TX 78712

W. E. Drummond University of Texas Department of Physics Alistin, TX 78712

Abraham Hertzberg University of Washington Aerospace Research

Laboratory

316 Guggenhe im

Seattle, WA 98105

A. L. Babb

University of Washington

Nuclear Engineering

Department

Seattle, WA 98105

R. Conn

University of Wisconsin

Nuclear Engineering

Department

Madison, WI 53706

G. L. Kulcinski

University of Wisconsin

Nuclear Engineering

Department

Madison, WI 53706

C. W. Maynard

University of Wisconsin

Nuclear Engineering

Department

Madison, WI 53706

D. Lichtman

Department of Physics

University of Wisconsin

Milwaukee, WE 53201

E. E. Donaldson Washington State University

Deparment of Physics

Pullman, WA 99163

D. D. Mahlum

Division of Biomedical

and Environmental Research

Washington, DC 20545

J. V. Vanston

Engineering Science

Building

University of Texas

Austin, TX 78712

Leslie S. Ramsey

450 North 5 th Street

Indiana, PA 15701 
ERDA Richland Operations Office

W. A. Burns

Atlantic Richfield Hanford Company

J. D. Kaser

Hanford Engineering Development Labs

D. G. Doran

H. H. Yoshikawa

\section{Battelle-Northwest}

D. T. Aase

G. S. Allison

T. W. Ambrose

o. G. Atteridge

D. A. Baker

J. L. Bates

M. A. Bayne

E. R. Bradley

J. L. Brimhal 1

R. L. Brodzinski

R. J. Brouns

L. R. Bunnell

L. L. Burger

S. H. Bush

N. E. Carter

T. D. Chikalla

R. G. Clark

T. L. Criswell

S. D. Dahlgren

M. T. Dana

D. E. Deonigi

R. L. Dillon

D. A. Dingee

P. J. Dionne

B. H. Duare

J. W. Finnigan

J. C. Fox

J. J. Fuquay

J. E. Garnier

R. D. Gastil

10 B. F. Gore

J. N. Hartley

A. J. Haverfield

U. P. Jenquin

A. B. Johnson, Jr.

R. H. Jones

T. J. Kabele

W. S. Kelly

H. E. Kissinger

D. A. Kottwitz

N. Laegried

B. R. Leonard, Jr

D. L. Lessor

H. B. Liemohn

R. C. Lijkala

M. A. Mckinnon

R. F. Maness

R. P. Marshall
Battelle-Northwest - Continued

E. S. Murphy

R. D. Nelson

D. F. Newman

R. E. Nightingale

O. E. Olesen

L. T. Pedersen

R. T. Perry

D. R. Pratt

L. A. Rancitelli

J. F. Remark

U. S. Renne

R. E. Rhoads

W. D. Richmond

W. F. Sandusky

L. C. Schmid

iv. M. Sherer

E. P. Simonen

R. I. Smith

J. K. Soldat

C. W. Stewart

K. B. Stewart

R. W. Stewart

J. A. Strand

D. L. Styris

A. M. Sutey

V. L. Teofilo

G. L. Tingey

M. T. Thomas

R. C. Thompson

$L$. H. Toburen

T. J. Trapp

R. Wang

R. E: Westerman

L. D. Williams

20 J. R. Young

M. G. Zimmerman

1 Technical Publications (BH)

5 Technical Information 